\title{
ASYMPTOTIC BEHAVIOR OF THE HITTING TIME, OVERSHOOT AND UNDERSHOOT FOR SOME LÉVY PROCESSES
}

\author{
Bernard Roynette ${ }^{1},{\text { Pierre } \text { VAllois }^{1} \text { And Agnès Volpi }}^{1,2}$
}

\begin{abstract}
Let $\left(X_{t}, t \geq 0\right)$ be a Lévy process started at 0 , with Lévy measure $\nu$. We consider the first passage time $T_{x}$ of $\left(X_{t}, t \geq 0\right)$ to level $x>0$, and $K_{x}:=X_{T_{x}}-x$ the overshoot and $L_{x}:=x-X_{T_{x}-}$ the undershoot. We first prove that the Laplace transform of the random triple $\left(T_{x}, K_{x}, L_{x}\right)$ satisfies some kind of integral equation. Second, assuming that $\nu$ admits exponential moments, we show that $\left(\widetilde{T_{x}}, K_{x}, L_{x}\right)$ converges in distribution as $x \rightarrow \infty$, where $\widetilde{T_{x}}$ denotes a suitable renormalization of $T_{x}$.
\end{abstract}

Mathematics Subject Classification. 60E10, 60F05, 60G17, 60G40, 60G51, 60J65, 60J75, 60J80, 60K05.

Received February 16, 2007.

\section{INTRODUCTION}

1. Let $\left(X_{t}, t \geq 0\right)$ be a Lévy process, which is right continuous with left limits and starts at 0 . Let

$$
X_{t}=\sigma B_{t}-c_{0} t+J_{t} \quad t \geq 0
$$

be the canonical decomposition, when $c_{0} \in \mathbb{R}, \sigma>0$ and $\left(B_{t}, t \geq 0\right)$ is a one-dimensional Brownian motion started at 0 . $\left(J_{t}, t \geq 0\right)$ is a pure jump Lévy process which is independent from $\left(B_{t}, t \geq 0\right)$. In addition $J_{0}=0$. Recall (see for instance Th. 2.1, Chap. 2, [13]) that $\left(J_{t}, t \geq 0\right)$ is the sum of a compound Poisson process and a square integrable martingale whose jumps are of magnitude less than 1 .

For simplicity, we may assume that $\sigma=1$.

2. We are interested in the first hitting time of level $x>0$

$$
T_{x}:=\inf \left\{t \geq 0 ; X_{t}>x\right\}
$$

Keywords and phrases. Lévy processes, ruin problem, hitting time, overshoot, undershoot, asymptotic estimates, functional equation.

1 Département de mathématiques, Institut Élie Cartan,Université Henri Poincaré, BP 239, 54506 Vandouvre-lès-Nancy cedex, France; [roynette; vallois] @iecn.u-nancy.fr

2 ESSTIN, 2 rue Jean Lamour, Parc Robert Bentz, 54500 Vandœuvre-lès-Nancy, France; volpi@esstin.uhp-nancy.fr 
We also consider the overshoot $K_{x}$ and the undershoot $L_{x}$ :

$$
\begin{aligned}
& K_{x}:=X_{T_{x}}-x, \\
& L_{x}:=x-X_{T_{x-}} .
\end{aligned}
$$

The aim of this paper is to study the joint distribution of the triple $\left(T_{x}, K_{x}, L_{x}\right)$.

3. In the usual theory of risk in continuous time the surplus of an insurance company is modelled by a stochastic process $\left(Z_{t}, t \geq 0\right)$. The real number $x=Z_{0}$ denotes the initial surplus, and the random time $T_{x}:=\inf \left\{t \geq 0 ; Z_{t}<0\right\}$ may be interpreted as the ruin time. Historically, the first model (called classical or the Cramér-Lundberg one) was initiated by Lundberg [16] and Cramér [3,4]. It refers to the case when $\left(Z_{t}, t \geq 0\right)$ is the sum of a drift and a compound Poisson process. The later represents the aggregate claims. In [8], Dufresne and Gerber have added a Brownian perturbation in the surplus process. Thus, the process $\left(x-Z_{t}, t \geq 0\right)$ corresponds to a particular case of our process $\left(X_{t}, t \geq 0\right)$. A lot of authors have developed extensions and have considered a great variety of processes $\left(Z_{t}, t \geq 0\right)$. They have mainly focused on the choice of more adapted processes $\left(Z_{t}, t \geq 0\right)$ to take into account the complex reality. It is not our purpose to present here all these developments. For more information one should refer to Rolski, Schmidli, Schmidt and J. Teugels' book [17], in which a large panel of models can be found.

Here are a few papers which are closely connected to the limit distribution of $\left(T_{x}, K_{x}, L_{x}\right)$, as $x \rightarrow \infty$.

(a) Bertoin and Doney in [2] proved that the ruin probability $\mathbb{P}\left(T_{x}<\infty\right)$ is equivalent to $C \mathrm{e}^{-\alpha x}$, as $x \rightarrow \infty$. The authors has given in their paper an expression of the constant $C$ with the ascending ladder height process associated with $\left(X_{t}, t \geq 0\right)$. When the Lévy process $\left(X_{t}, t \geq 0\right)$ has no negative jumps, then $C$ and $\alpha$ may be calculated explicitly.

(b) In the discrete time model, i.e. when $\left(X_{t}, t \geq 0\right)$ is replaced by a random walk, Gut [11] has considered the limit distribution of normalized passage times.

(c) In both [10] and [7] analytical conditions are given to ensure that the ratio $X_{T_{x}} / x$ almost surely converges to 1 , as $x \rightarrow \infty$, in the discrete time model as well as in the case of Lévy processes.

(d) Doney and Kyprianou [6] have showed that $\left(K_{x}, L_{x}\right)$ converges in the distribution sense as $x$ goes to infinity.

Our study presents the following features:

(a) original analytic arguments of complex analysis are used, and especially meromorphic and holomorphic functions (see Th. 2.8);

(b) a decorrelation phenomenon: the couple $\left(K_{x}, L_{x}\right)$ and a relevant normalization of $T_{x}$ become asymptotically independent, $(x \rightarrow \infty)$ (cf. Ths. 2.1 and 2.3$)$;

(c) new functional equations (cf. Ths. 2.4 and 2.5).

4. Let us briefly describe the organization of the paper. In section 1, we will set up notation and assumptions. In Section 2, we will suppose that the Lévy measure $\nu$ of $\left(X_{t}, t \geq 0\right)$ satisfies the condition $(H)$, which is defined in item 1.4 of Section 1.

In Section 2 we will list the main results of the paper. The two major theorems (cf. Ths. 2.1 and 2.3 ) are related to the convergence in distribution of the triple $\left(\widetilde{T_{x}}, K_{x}, L_{x}\right)$ with $x \rightarrow \infty$. In addition $\widetilde{T_{x}}$ is expressed in terms of $T_{x}$ and $x$ and it depends on the sign of $\mathbb{E}\left(X_{1}\right)$. In Section 2.2 we present the important theorems which permit to demonstrate Theorems 2.1 and 2.3. Our approach is based on the study of the Laplace transform $F$ of $\left(T_{x}, K_{x}, L_{x}\right)$ :

$$
F(\theta, \mu, \rho, x):=\mathbb{E}\left(\mathrm{e}^{-\theta T_{x}-\mu K_{x}-\rho L_{x}} \mathbb{1}_{\left\{T_{x}<\infty\right\}}\right), \quad \theta, \mu, \rho \geq 0
$$


When $\nu(\mathbb{R})$ is finite, it is shown in Theorem 2.4 that $F(\theta, \mu, \rho, \cdot)$ satisfies some kind of integral equation. Introducing the Laplace transform $\widehat{F}(\theta, \mu, \rho, \cdot)$ of $F(\theta, \mu, \rho, \cdot)$ :

$$
\widehat{F}(\theta, \mu, \rho, q):=\int_{0}^{+\infty} \mathrm{e}^{-q y} F(\theta, \mu, \rho, y) \mathrm{d} y
$$

we proved in Theorem 2.5 that, under $(H)$, the function $\widehat{F}(\theta, \mu, \rho, \cdot)$ solves an equation which looks like an integral equation. Then an asymptotic development of $F(\theta, \mu, \rho, x)(x \rightarrow \infty)$ with a finite number of terms like $C(\theta, \mu, \rho) \mathrm{e}^{-\alpha(\theta) x}$ is given in Theorem 2.8. In the third Section 2.3 we will determine the behavior of the ruin probability $\mathbb{P}\left(T_{x}<\infty\right)$, when $x$ runs to infinity. Theorem 2.10 asserts that the ruin probability has a polynomial rate of decay at $\infty$ as soon as $\nu$ admits polynomial moments. All the proofs of results stated in Section 2 are postponed in Section 3.

Finally, in the last Section 4 we will give some complements and comments.

\section{Characteristic exponent and elementary properties}

1.1 Let $\psi$ be the characteristic exponent of a Lévy process $\left(X_{t}, t \geq 0\right)$ with canonical decomposition (0.1), i.e. $\mathbb{E}\left(\mathrm{e}^{i q X_{t}}\right)=\mathrm{e}^{t \psi(q)}(q \in \mathbb{R})$. With the Lévy-Khintchine formula, we get:

$$
\psi(q)=-\frac{q^{2}}{2}-i c q+\int_{\mathbb{R}}\left(\mathrm{e}^{i q y}-1-i q y \mathbb{1}_{\{|y|<1\}}\right) \nu(\mathrm{d} y), \quad q \in \mathbb{R}
$$

where $\nu$ is the Lévy measure which satisfies $\int_{\mathbb{R}}\left(y^{2} \wedge 1\right) \nu(\mathrm{d} y)<\infty$.

It is well known (cf. [18], example 25.12) that as soon as $\int_{\mathbb{R}}|y| \mathbb{1}_{\{|y| \geq 1\}} \nu(\mathrm{d} y)<\infty$ then

$$
\mathbb{E}\left[\left|X_{1}\right|\right]<\infty
$$

and

$$
\mathbb{E}\left(X_{1}\right)=-i \psi^{\prime}(0)=-c+\int_{\mathbb{R}} y \mathbb{1}_{\{|y| \geq 1\}} \nu(\mathrm{d} y) .
$$

When $\left(J_{t}, t \geq 0\right)$ is a compound Poisson process, there exists a relation between the drift term $c_{0}$ in $(0.1)$ and $c$ :

$$
c=c_{0}-\int_{\mathbb{R}} y \mathbb{1}_{\{|y|<1\}} \nu(\mathrm{d} y) .
$$

1.2 The following assumptions will be needed throughout the paper:

$$
\left.\int_{-\infty}^{-1} \mathrm{e}^{s y} \nu(\mathrm{d} y)<\infty, \forall s \in\right]-\infty, 0[
$$

and

It is convenient to introduce:

$$
\int_{1}^{\infty} \mathrm{e}^{s y} \nu(\mathrm{d} y)<\infty, \quad \text { for some } s>0
$$

$$
r_{\nu}:=\sup \left\{s \geq 0 ; \int_{1}^{\infty} \mathrm{e}^{s y} \nu(\mathrm{d} y)<\infty\right\} .
$$

It is clear that (1.12) implies that $\left.\left.r_{\nu} \in\right] 0, \infty\right]$. 
Under (1.11) and (1.12), the function $\psi$ may be extended to the half-space $\left\{z \in \mathbb{C} ; \operatorname{Im}(z)>-r_{\nu}\right\}$. Let $\varphi$ denote the function: $\varphi(q):=\psi(i q), q>-r_{\nu}$. If we consider the definition of $\psi$ and the identity (1.7), we can infer that:

$$
\mathbb{E}\left(\mathrm{e}^{-q X_{t}}\right)=\mathrm{e}^{t \varphi(q)}
$$

and

for any $q \in]-r_{\nu}, \infty[$.

$$
\varphi(q)=\frac{q^{2}}{2}+c q+\int_{\mathbb{R}}\left(\mathrm{e}^{-q y}-1+q y \mathbb{1}_{\{|y|<1\}}\right) \nu(\mathrm{d} y)
$$

Note that:

$$
\varphi^{\prime}(0)=-\mathbb{E}\left(X_{1}\right)
$$

Assumptions (1.11) and (1.12) imply that $\varphi$ is a function defined on $]-r_{\nu}, \infty[$ and:

$$
\left.\varphi^{\prime \prime}(q)=1+\int_{\mathbb{R}} y^{2} \mathrm{e}^{-q y} \nu(\mathrm{d} y), \quad \text { for any } q \in\right]-r_{\nu}, \infty[
$$

Consequently, $\varphi$ is convex on ] $-r_{\nu}, \infty\left[\right.$. It can be easily shown that $\varphi(\infty):=\lim _{x \rightarrow \infty} \varphi(x)=\infty$.

Let us discuss the behavior of $\varphi$ in the vicinity of $-r_{\nu}$.

1. When $r_{\nu}=\infty$ then $\varphi\left(-r_{\nu}\right):=\lim _{q \rightarrow \infty} \varphi(q)=\infty$.

2. When $r_{\nu}<\infty$ and $\int_{1}^{\infty} \mathrm{e}^{r_{\nu} y} \nu(\mathrm{d} y)=\infty$ then $\varphi\left(-r_{\nu}\right):=\lim _{q \rightarrow-r_{\nu}} \varphi(q)=\infty$.

3. In the case:

$$
r_{\nu}<\infty \text { and } \int_{1}^{\infty} \mathrm{e}^{r_{\nu} y} \nu(\mathrm{d} y)<\infty,
$$

then $\varphi\left(-r_{\nu}\right)$ is a real number and:

$$
\varphi\left(-r_{\nu}\right)=\frac{r_{\nu}^{2}}{2}-c r_{\nu}+\int_{\mathbb{R}}\left(\mathrm{e}^{r_{\nu} y}-1-r_{\nu} y \mathbb{1}_{\{|y|<1\}}\right) \nu(\mathrm{d} y) .
$$

Let $c_{\nu}$ be the real number defined as:

$$
\frac{r_{\nu}^{2}}{2}-c_{\nu} r_{\nu}+\int_{\mathbb{R}}\left(\mathrm{e}^{r_{\nu} y}-1-r_{\nu} y \mathbb{1}_{\{|y|<1\}}\right) \nu(\mathrm{d} y)=0
$$

Hence:

$$
\varphi\left(-r_{\nu}\right)>0 \Leftrightarrow c<c_{\nu}
$$

In the rest of the paper we assume

$$
\left.\left.\varphi\left(-r_{\nu}\right) \in\right] 0, \infty\right]
$$

holds in any case.

1.3 In the sequel of the paper it will be convenient to deal with the parameter $\mathbb{E}\left(X_{1}\right)$. Note that this expectation may be expressed in terms of $c$ and $\nu$, via (1.9).

The zeros of

$$
\varphi_{\theta}(.):=\varphi(.)-\theta
$$

are important parameters of our study. We can plot two graphs:

(1) one represents $\varphi$ and corresponds respectively to the three cases: $\mathbb{E}\left(X_{1}\right)<0, \mathbb{E}\left(X_{1}\right)>0$ and $\mathbb{E}\left(X_{1}\right)=0$;

(2) another which represents $\varphi_{\theta}$;

(see Figs. 1 and 2 for illustration). 


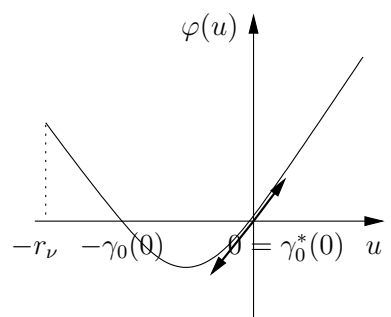

a) $\mathbb{E}\left(X_{1}\right)=-\varphi^{\prime}(0)<0$

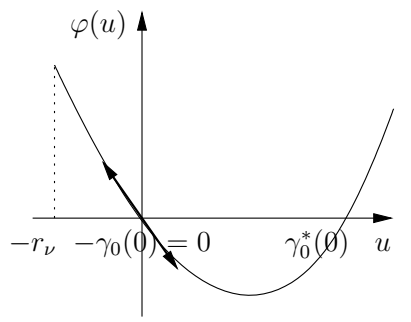

b) $\mathbb{E}\left(X_{1}\right)=-\varphi^{\prime}(0)>0$

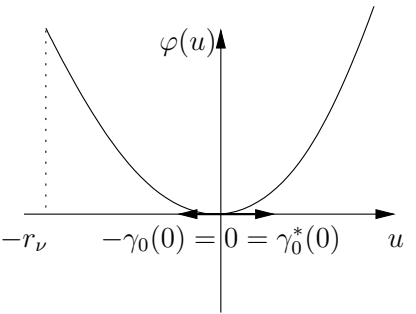

c) $\mathbb{E}\left(X_{1}\right)=-\varphi^{\prime}(0)=0$

FiguRE 1. Graph of $\varphi$.

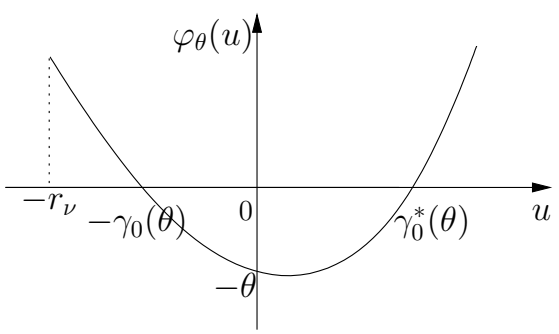

FiguRE 2. Graph of $\varphi_{\theta}$.

From Figures 1 and 2, we can easily infer the existence of $\kappa>0$ so that:

1. there is a unique $\gamma_{0}^{*}(\theta) \geq 0$ which satisfies:

$$
\varphi\left(\gamma_{0}^{*}(\theta)\right)=\theta, \quad \forall \theta \in[0, \kappa]
$$

and

$$
\gamma_{0}^{*}(\theta) \begin{cases}>0 & \text { if } \theta>0, \text { or } \theta=0 \text { and } \mathbb{E}\left(X_{1}\right)>0 \\ =0 & \text { if } \theta=0 \text { and } \mathbb{E}\left(X_{1}\right) \leq 0\end{cases}
$$

2. there is a unique $\gamma_{0}(\theta) \geq 0$ which satisfies:

$$
\varphi\left(-\gamma_{0}(\theta)\right)=\theta, \quad \forall \theta \in[0, \kappa]
$$

and

$$
-\gamma_{0}(\theta) \begin{cases}<0 & \text { if } \theta>0, \text { or } \theta=0 \text { and } \mathbb{E}\left(X_{1}\right)<0 \\ =0 & \text { if } \theta=0 \text { and } \mathbb{E}\left(X_{1}\right) \geq 0\end{cases}
$$

So, when $\theta>0$ is rather small, the positive (resp. negative) zero of $\varphi_{\theta}$ is $\gamma_{0}^{*}(\theta)$ (resp. $-\gamma_{0}(\theta)$ ).

1.4 In the rest of the paper, excepted in Section 2.3, we will require that $\nu$ and $c$ satisfy (1.11), (1.12) and (1.21). On principle, let us consider these three conditions as as- sumption $(H)$.

Note that, under $(H)$, there is $\kappa>0$ so that $(1.23)-(1.26)$ holds. 


\section{The Results}

We keep notation given in Section 1.

\subsection{Normalized limit distribution of $\left(T_{x}, K_{x}, L_{x}\right)$, as $x \rightarrow \infty$}

In this section we will investigate the limit behavior of the triple $\left(T_{x}, K_{x}, L_{x}\right)$, as $x \rightarrow \infty$. Recall that $K_{x}$ and $L_{x}$ are defined by (0.3), resp. (0.4). Here as three cases: either $\mathbb{E}\left(X_{1}\right)>0$, or $\mathbb{E}\left(X_{1}\right)<0$ or $\mathbb{E}\left(X_{1}\right)=0$. First, it can be assumed that $\mathbb{E}\left(X_{1}\right)<0$.

Theorem 2.1. Under $(H)$ and $\mathbb{E}\left(X_{1}\right)<0$ then, conditionally on $\left\{T_{x}<\infty\right\}$, the triple $\left(\frac{1}{\sqrt{x}}\left(T_{x}+\frac{x}{\varphi^{\prime}\left(-\gamma_{0}(0)\right)}\right), K_{x}, L_{x}\right)$ converges in distribution when $x \rightarrow \infty$ to the 3-dimensional law $\mathcal{N}\left(0 ;-\frac{\varphi^{\prime \prime}\left(-\gamma_{0}(0)\right)}{\varphi^{\prime 3}\left(-\gamma_{0}(0)\right)}\right) \otimes w^{-}$. In addition, $w^{-}$is the probability measure on $\mathbb{R}_{+} \times \mathbb{R}_{+}$:

$$
\begin{aligned}
w^{-}(\mathrm{d} k, \mathrm{~d} l)= & \frac{-1}{\mathbb{E}\left(X_{1}\right)}\left[\frac{\gamma_{0}(0)}{2} \delta_{0,0}(\mathrm{~d} k, \mathrm{~d} l)+\left(\mathrm{e}^{\gamma_{0}(0) l}-1\right) \mathbb{1}_{\{k \geq 0 ; l \geq 0\}} \nu_{l}(\mathrm{~d} k) \mathrm{d} l\right. \\
& \left.+\int_{\mathbb{R}_{-}}\left(\int_{0}^{-y}\left(1-\mathrm{e}^{\gamma_{0}(0)(b+y)}\right) \mathbb{P}\left(T_{b}<\infty\right) n(b, \mathrm{~d} k, \mathrm{~d} l) \mathrm{d} b \mathbb{1}_{\{k \geq 0, l \geq 0\}}\right) \nu(\mathrm{d} y)\right]
\end{aligned}
$$

$\nu_{l}(\mathrm{~d} k)$ is the image of $\nu(\mathrm{d} k)$ by the map $y \rightarrow y-l$, and $n(b, \mathrm{~d} k, \mathrm{~d} l)$ is the distribution of $\left(K_{b}, L_{b}\right)$ conditionally on $\left\{T_{b}<\infty\right\}$.

If moreover the support of $\nu$ is included in $\left[0 ; \infty\left[, w^{-}(\mathrm{d} k, \mathrm{~d} l)\right.\right.$ is given explicitly:

$$
w^{-}(\mathrm{d} k, \mathrm{~d} l)=\frac{-1}{\mathbb{E}\left(X_{1}\right)}\left[\frac{\gamma_{0}(0)}{2} \delta_{0,0}(\mathrm{~d} k, \mathrm{~d} l)+\left(\mathrm{e}^{\gamma_{0}(0) l}-1\right) \mathbb{1}_{\{k \geq 0 ; l \geq 0\}} \nu_{l}(\mathrm{~d} k) \mathrm{d} l\right] .
$$

\section{Remark 2.2.}

1. $\mathcal{N}\left(0 ; \sigma^{2}\right)$ denotes the Gaussian distribution with mean 0 and variance $\sigma^{2}$.

2. a) We may observe that time and positions become asymptotically independent. However the two components of the positions are not independent. In Section 4.2, we give a stochastic interpretation of the limit distribution $w^{-}(\mathrm{d} k, \mathrm{~d} l)$ defined by $(2.2)$.

b) Obviously $K_{x}+L_{x}=X_{T_{x}}-X_{T_{x}}$ is the jump size of $\left(X_{t}, t \geq 0\right)$ at $T_{x}$. It is easy to infer from Theorem 2.1 that $\left(\frac{1}{\sqrt{x}}\left(T_{x}+\frac{x}{\varphi^{\prime}\left(-\gamma_{0}(0)\right)}\right), X_{T_{x}}-X_{T_{x}}{ }^{-}\right)$converges in distribution, as $x \rightarrow \infty$, to $\mathcal{N}\left(0 ;-\frac{\varphi^{\prime \prime}\left(-\gamma_{0}(0)\right)}{\varphi^{\prime 3}\left(-\gamma_{0}(0)\right)}\right) \otimes \widetilde{w} \quad$ where $\widetilde{w}$ is the probability measure on $\mathbb{R}_{+}$:

$$
\begin{aligned}
\widetilde{w}(\mathrm{~d} s)= & \frac{-1}{\mathbb{E}\left(X_{1}\right)}\left[\frac{\gamma_{0}(0)}{2} \delta_{0}(\mathrm{~d} s)+\frac{\mathrm{e}^{\gamma_{0}(0) s}-1-\gamma_{0}(0) s}{\gamma_{0}(0)} \mathbb{1}_{\{s \geq 0\}} \nu(\mathrm{d} s)\right. \\
& \left.+\int_{\mathbb{R}_{-}}\left(\int_{0}^{-y}\left(1-\mathrm{e}^{\gamma_{0}(0)(b+y)}\right) \mathbb{P}\left(T_{b}<\infty\right) n(b, \mathrm{~d} s) \mathrm{d} b\right) \nu(\mathrm{d} y) \mathbb{1}_{\{s \geq 0\}}\right],
\end{aligned}
$$

and $n(b, \mathrm{~d} s)$ is the distribution of $X_{T_{b}}-X_{T_{b}}$ - conditionally on $\left\{T_{b}<\infty\right\}$.

If the jumps of $\left(X_{t}, t \geq 0\right)$ are positive, $\widetilde{w}(\mathrm{~d} s)$ can be simplified:

$$
\widetilde{w}(\mathrm{~d} s)=\frac{-1}{\mathbb{E}\left(X_{1}\right)}\left[\frac{\gamma_{0}(0)}{2} \delta_{0}(\mathrm{~d} s)+\frac{\mathrm{e}^{\gamma_{0}(0) s}-1-\gamma_{0}(0) s}{\gamma_{0}(0)} \mathbb{1}_{\{s \geq 0\}} \nu(\mathrm{d} s)\right] .
$$

Let list the results related to the two other cases: $\mathbb{E}\left(X_{1}\right)>0$ and $\mathbb{E}\left(X_{1}\right)=0$. 
Theorem 2.3. Assume $(H)$.

1. When $\mathbb{E}\left(X_{1}\right)>0$, then $T_{x}<\infty$ a.s. and the triple $\left(\frac{1}{\sqrt{x}}\left(T_{x}+\frac{x}{\varphi^{\prime}(0)}\right), K_{x}, L_{x}\right)$ converges in distribution, as $x \rightarrow \infty$, to $\mathcal{N}\left(0 ;-\frac{\varphi^{\prime \prime}(0)}{\varphi^{\prime 3}(0)}\right) \otimes w^{+}$, where $w^{+}$is defined by the relation obtained after replacing $\gamma_{0}(0)$ by $-\gamma_{0}^{*}(0)$ in (2.1).

In particular if $\left(X_{t}, t \geq 0\right)$ has only positive jumps:

$$
w^{+}(\mathrm{d} k, \mathrm{~d} l)=\frac{1}{\mathbb{E}\left(X_{1}\right)}\left[\frac{\gamma_{0}^{*}(0)}{2} \delta_{0,0}(\mathrm{~d} k, \mathrm{~d} l)+\left(1-\mathrm{e}^{-\gamma_{0}^{*}(0) l}\right) \mathbb{1}_{\{k \geq 0, l \geq 0\}} \nu_{l}(\mathrm{~d} k) \mathrm{d} l\right] .
$$

2. When $\mathbb{E}\left(X_{1}\right)=0$, then $\left(\frac{T_{x}}{x^{2}}, K_{x}, L_{x}\right)$ converges in distribution, as $x \rightarrow \infty$, to $\varrho \otimes w^{0}$. $\varrho$ denotes the law of the first hitting time of level $\sqrt{\frac{1}{\varphi^{\prime \prime}(0)}}$ by a standard Brownian motion started at 0 . Let us recall that $\int_{0}^{\infty} \mathrm{e}^{-\theta x} \varrho(\mathrm{d} x)=\mathrm{e}^{-\sqrt{\frac{2 \theta}{\varphi^{\prime \prime}(0)}}}$. Moreover $\varphi^{\prime \prime}(0)=1+\int_{\mathbb{R}} y^{2} \nu(\mathrm{d} y)$

The probability measure $w^{0}$ on $\mathbb{R}_{+} \times \mathbb{R}_{+}$is defined as follows:

$$
\begin{aligned}
w^{0}(\mathrm{~d} k, \mathrm{~d} l)= & \frac{1}{\varphi^{\prime \prime}(0)}\left[\delta_{0,0}(\mathrm{~d} k, \mathrm{~d} l)+2 l \mathbb{1}_{\{k \geq 0, l \geq 0\}} \nu_{l}(\mathrm{~d} k) \mathrm{d} l\right. \\
& \left.-2 \int_{\mathbb{R}_{-}}\left(\int_{0}^{-y}(b+y) n(b, \mathrm{~d} k, \mathrm{~d} l) \mathrm{d} b \mathbb{1}_{\{k \geq 0, l \geq 0\}}\right) \nu(\mathrm{d} y)\right],
\end{aligned}
$$

when $n(b, \mathrm{~d} k, \mathrm{~d} l)$ is the distribution of $\left(K_{b}, L_{b}\right)$.

This expression may be simplified if $\left(X_{t}, t \geq 0\right)$ has only positive jumps (i.e. $\left.\nu(]-\infty, 0[)=0\right)$ :

$$
w^{0}(\mathrm{~d} k, \mathrm{~d} l)=\frac{1}{\varphi^{\prime \prime}(0)}\left[\delta_{0,0}(\mathrm{~d} k, \mathrm{~d} l)+2 l \mathbb{1}_{\{k \geq 0, l \geq 0\}} \nu_{k}(\mathrm{~d} l) \mathrm{d} k\right]
$$

We will present two complements of Theorems 2.1 and 2.3 .

1. Let us introduce:

$$
\widetilde{T}_{x}=\left\{\begin{array}{cc}
\frac{1}{\sqrt{x}}\left(T_{x}+\frac{x}{\varphi^{\prime}\left(-\gamma_{0}(0)\right)}\right) & \text { when } \mathbb{E}\left(\mathrm{X}_{1}\right)<0 \\
\frac{1}{\sqrt{x}}\left(T_{x}+\frac{x}{\varphi^{\prime}(0)}\right) & \text { when } \mathbb{E}\left(\mathrm{X}_{1}\right)>0 \\
\frac{T_{x}}{x^{2}} & \text { when } \mathbb{E}\left(\mathrm{X}_{1}\right)=0
\end{array}\right.
$$

In Section 4.1, a rate of convergence of $\widetilde{T}_{x}$ to the associated Gaussian distribution, as $x \rightarrow \infty$ is given.

2. In Section 4.2, we will provide a stochastic realization of the probability measure $w^{-}$, resp. $w^{+}$defined by $(2.2)$, resp. $(2.5)$.

\subsection{Auxiliary results}

To study the joint distribution of $\left(T_{x}, K_{x}, L_{x}\right)$, the Laplace transform $F$ of this three dimensional r.v. is used:

$$
F(\theta, \mu, \rho, x)=\mathbb{E}\left(\mathrm{e}^{-\theta T_{x}-\mu K_{x}-\rho L_{x}} \mathbb{1}_{\left\{T_{x}<\infty\right\}}\right)
$$

for any $\theta \geq 0, \mu \geq 0, \rho \geq 0$.

a) When $\nu(\mathbb{R})<\infty$ (i.e. $\left(J_{t}, t \geq 0\right)$ is a compound Poisson process), we first prove (see Th. 2.4 below) that $F(\theta, \mu, \rho, \cdot)$ satisfies a kind of integral equation. 
Theorem 2.4. Assume $\lambda=\nu(\mathbb{R})<\infty$. For any $\theta \geq 0, \mu \geq 0$ and $\rho \geq 0$, the function $F(\theta, \mu, \rho,$.$) is solution$ of the following integral equation:

$$
G(x)=F_{0}(\theta, \mu, \rho, x)+F_{1}(\theta, \mu, \rho, x)+\Lambda_{\theta} G(x) \quad \forall x \geq 0
$$

where

$$
\begin{aligned}
\alpha_{\theta}= & \sqrt{c_{0}^{2}+2(\lambda+\theta)}, \\
F_{0}(\theta, \mu, \rho, x)= & \mathrm{e}^{-\left(c_{0}+\alpha_{\theta}\right) x}, \\
F_{1}(\theta, \mu, \rho, x)= & \frac{\mathrm{e}^{-\left(c_{0}+\alpha_{\theta}\right) x}}{\alpha_{\theta}\left(\mu-\rho+c_{0}+\alpha_{\theta}\right)} \int_{[0, x]}\left(\mathrm{e}^{\left(-\rho+c_{0}+\alpha_{\theta}\right) y}-\mathrm{e}^{-\mu y}\right) \nu(\mathrm{d} y) \\
& +\frac{\mathrm{e}^{-\rho x}}{\alpha_{\theta}\left(\mu-\rho+c_{0}-\alpha_{\theta}\right)} \int_{] x, \infty[}\left(\mathrm{e}^{-\left(\rho+\alpha_{\theta}-c_{0}\right)(y-x)}-\mathrm{e}^{-\mu(y-x)}\right) \nu(\mathrm{d} y) \\
& +\frac{\mathrm{e}^{(\mu-\rho) x}-\mathrm{e}^{-\left(c_{0}+\alpha_{\theta}\right) x}}{\alpha_{\theta}\left(\mu-\rho+c_{0}+\alpha_{\theta}\right)} \int_{] x, \infty[} \mathrm{e}^{-\mu y} \nu(\mathrm{d} y) \\
& -\frac{\mathrm{e}^{-\left(c_{0}+\alpha_{\theta}\right) x}}{\alpha_{\theta}\left(\mu-\rho+c_{0}-\alpha_{\theta}\right)} \int_{0}^{\infty}\left(\mathrm{e}^{-\left(\rho+\alpha_{\theta}-c_{0}\right) y}-\mathrm{e}^{-\mu y}\right) \nu(\mathrm{d} y),
\end{aligned}
$$

and $\Lambda_{\theta}$ is the operator:

$$
\Lambda_{\theta} G(x)=\frac{1}{\alpha_{\theta}} \int_{-\infty}^{\infty} \nu(\mathrm{d} y) \int_{-\infty}^{(x-y) \wedge x} \mathrm{e}^{-c_{0} a}\left(\mathrm{e}^{-\alpha_{\theta}|a|}-\mathrm{e}^{-(2 x-a) \alpha_{\theta}}\right) G(x-a-y) \mathrm{d} a .
$$

The positive operator $\Lambda_{\theta}$ will be studied in details in Section 4.3. In particular, it is proved that, under suitable assumptions, $F(\theta, \mu, \rho, \cdot)$ is the unique function $G$ which solves the equation (2.10), and can be strongly approximated by a series.

b) However the operator $\Lambda_{\theta}$ cannot be defined if $\nu$ is not a finite measure. The formula (2.10) does not permit to consider Lévy processes which are not reduced to a Brownian motion with drift plus a compound Poisson process. To avoid this difficulty, we introduce the Laplace transform $\widehat{F}(\theta, \mu, \rho, \cdot)$ of $F(\theta, \mu, \rho, \cdot)$ :

$$
\widehat{F}(\theta, \mu, \rho, q)=\int_{0}^{\infty} \mathrm{e}^{-q y} F(\theta, \mu, \rho, y) \mathrm{d} y
$$

This definition is meaningful for any $q$ such that $\operatorname{Re}(q)>0$, since $F(\theta, \mu, \rho,$.$) is a bounded function on [0, \infty[$.

Taking the Laplace transform in (2.10), proves (see Th. 2.5 below) that under $(H), \widehat{F}(\theta, \mu, \rho,$.$) verifies some$ kind of integral equation which remains valid when $\nu$ is a Lévy measure with no necessary finite mass. Before stating this result, let us denote by $R$ the operator:

$$
R h(q):=\int_{-\infty}^{0} \nu(\mathrm{d} y) \int_{0}^{-y}\left(\mathrm{e}^{-q(b+y)}-1\right) h(b) \mathrm{d} b
$$

where $q \in \mathbb{C}, \operatorname{Re}(q)>0$ and $h \in L^{\infty}\left(\mathbb{R}_{+}\right)$.

Note that the identity (1.11) implies that $R h(q)$ is well defined. 
Theorem 2.5. Let us assume that $(H)$ holds. Let $\theta, \mu, \rho \geq 0, q \in \mathbb{C}, \operatorname{Re}(q)>0$. We get:

$$
\begin{aligned}
\widehat{F}(\theta, \mu, \rho, q) & =\frac{1}{\varphi(q)-\theta}\left(\frac{q-\gamma_{0}^{*}(\theta)}{2}+\int_{0}^{\infty}\left[\frac{\mathrm{e}^{-(q+\rho) y}-\mathrm{e}^{-\mu y}}{q+\rho-\mu}-\frac{\mathrm{e}^{-\left(\gamma_{0}^{*}(\theta)+\rho\right) y}-\mathrm{e}^{-\mu y}}{\gamma_{0}^{*}(\theta)+\rho-\mu}\right] \nu(\mathrm{d} y)\right. \\
& \left.+R F(\theta, \mu, \rho, .)(q)-R F(\theta, \mu, \rho, .)\left(\gamma_{0}^{*}(\theta)\right)\right)
\end{aligned}
$$

where $\gamma_{0}^{*}(\theta)$ is defined in (1.23) and (1.24).

\section{Remark 2.6.}

1. If $\nu(]-\infty, 0[)=0$ then $R F(\theta, \mu, \rho,$.$) is cancelled, and \widehat{F}(\theta, \mu, \rho, q)$ is given by the following explicit formula:

$$
\widehat{F}(\theta, \mu, \rho, q)=\frac{1}{\varphi(q)-\theta}\left(\frac{q-\gamma_{0}^{*}(\theta)}{2}+\int_{0}^{\infty}\left[\frac{\mathrm{e}^{-(q+\rho) y}-\mathrm{e}^{-\mu y}}{q+\rho-\mu}-\frac{\mathrm{e}^{-\left(\gamma_{0}^{*}(\theta)+\rho\right) y}-\mathrm{e}^{-\mu y}}{\gamma_{0}^{*}(\theta)+\rho-\mu}\right] \nu(\mathrm{d} y)\right) .
$$

2. If $\nu(] 0, \infty[)=0(2.17)$ is reduced to:

$$
\widehat{F}(\theta, \mu, \rho, q)=\frac{1}{\varphi(q)-\theta}\left(\frac{q-\gamma_{0}^{*}(\theta)}{2}+R F(\theta, \mu, \rho, .)(q)-R F(\theta, \mu, \rho, .)\left(\gamma_{0}^{*}(\theta)\right)\right)
$$

3. Let us briefly detail the case $\theta=\mu=\rho=0$ (i.e. $F(0,0,0, x)$ is the ruin probability). If $\mathbb{E}\left(X_{1}\right) \geq 0$, it is easy to check that $f: x \rightarrow 1$ satisfies (2.17). In the more interesting case: $\mathbb{E}\left(X_{1}\right)<0$, since $\gamma_{0}^{*}(0)=0$, then the relation (2.17) may be simplified. Suppose moreover that $\nu(]-\infty, 0[)=0$, then $(2.18)$ is reduced to:

$$
\widehat{F}(0,0,0, q)=\int_{0}^{\infty} \mathrm{e}^{-q y} \mathbb{P}\left(T_{y}<\infty\right) \mathrm{d} y=\frac{1}{q}+\frac{\mathbb{E}\left(X_{1}\right)}{\varphi(q)} .
$$

Therefore (2.20) generalizes identity (3.3) in [8].

Since $\left\{T_{y}<\infty\right\}=\left\{\bar{X}_{\infty}>y\right\}$, where $\bar{X}_{\infty}:=\sup _{t \geq 0} X_{t}$, then (2.20) is equivalent to:

$$
\mathbb{E}\left(\mathrm{e}^{-q \bar{X}_{\infty}}\right)=-\mathbb{E}\left(X_{1}\right) \frac{q}{\varphi(q)}, \quad q>0
$$

Note that in the case $\nu(] 0, \infty[)=0$, then $F(0,0,0, x)=\mathbb{P}\left(T_{x}<\infty\right)=\mathrm{e}^{-\gamma_{0}(0) x}$.

c) Let us briefly indicate how Theorem 2.5 enables to obtain the asymptotic behavior of $\left(\widetilde{T}_{x}, K_{x}, L_{x}\right)$ as $x \rightarrow$ $\infty$. Using the Mellin-Fourier inverse transformation, it is actually possible to recover $F(\theta, \mu, \rho, \cdot)$ (see the formula (3.70) in Sect. 3.4). It implies that the asymptotic behavior of $F(\theta, \mu, \rho, x), x \rightarrow \infty$ depends on the poles of $\widehat{F}(\theta, \mu, \rho, \cdot)$. According to (2.17), the complex zeros of $\varphi_{\theta}$ are the poles of $\widehat{F}(\theta, \mu, \rho, \cdot)$. Under $(H)$ and (2.23), we may determine in Proposition 2.7 the zeros of $\varphi_{\theta}$. Hence, we may obtain an expansion of $F(\theta, \mu, \rho, x)$ as $x \rightarrow \infty$ (see Th. 2.8 below). Finally that allows to determine the limit distribution of the random triple considered above as $x$ goes to infinity.

Let

$$
B_{\nu}:=\sup \left\{b>0 ; q \mapsto \int_{1}^{\infty} \mathrm{e}^{-q y} \nu(\mathrm{d} y) \text { admits a meromorphic extension to } D_{-b}\right\},
$$

where $D_{-b}:=\{q \in \mathbb{C} ; \operatorname{Re} q>-b\}$.

Note that $B_{\nu} \geq r_{\nu}$ and $B_{\nu}$ may be equal to $\infty$. Then $\varphi$ has a meromorphic extension to $D_{-} B_{\nu}$. For simplicity, this extension will be denoted $\varphi$. 
Our last assumption on $\nu$ is:

$$
\begin{aligned}
& \forall B \in] 0, B_{\nu}\left[, \exists K, R_{0}>0,\right. \text { so that : } \\
& \left|\int_{1}^{\infty} \mathrm{e}^{-q y} \nu(\mathrm{d} y)\right| \leq K|q|, \quad \text { for any } q \text { so that } \quad\left\{\begin{array}{c}
-B \leq \operatorname{Re} q \leq 0 \\
|\operatorname{Im} q| \geq R_{0}
\end{array}\right.
\end{aligned}
$$

A large class of measures $\nu$ satisfying $(H)$ and (2.23) will be given in Section 4.4 .

First, we will concentrate on the complex zeros of the function $\varphi_{\theta}$ defined by $(1.22)$. Then, we will give in Theorem 2.8 an asymptotic expansion of $F(\theta, \mu, \rho, x)$ as $x \rightarrow \infty$.

Proposition 2.7. Let us suppose that $(H)$ and (2.23) hold. Then for any $\theta \in[0, \kappa]$, there exists $\beta_{\theta}>0$ such that for any $B \in] 0, B_{\nu}\left[, \varphi_{\theta}\right.$ admits a finite number of conjugated zeros in the strip $D_{-B, \beta_{\theta}}:=\left\{q \in \mathbb{C} ;-B \leq \operatorname{Re} q \leq \beta_{\theta}\right\}$. This set of zeros of $\varphi_{\theta}$ in $D_{-B, \beta_{\theta}}$ is equal to (cf. Fig. 3 below):

1. $\left\{-\gamma_{0}(\theta),-\gamma_{1}(\theta),-\overline{\gamma_{1}}(\theta), \cdots,-\gamma_{p}(\theta),-\overline{\gamma_{p}}(\theta)\right\} \quad$ if $\theta>0$,

2. $\left\{0,-\gamma_{0}(0),-\gamma_{1}(0),-\overline{\gamma_{1}}(0), \cdots,-\gamma_{p}(0),-\overline{\gamma_{p}}(0)\right\} \quad$ if $\quad \theta=0$ and $\mathbb{E}\left(X_{1}\right)<0$,

3. $\left\{-\gamma_{0}(0)=0,-\gamma_{1}(0),-\overline{\gamma_{1}}(0), \cdots,-\gamma_{p}(0),-\overline{\gamma_{p}}(0)\right\} \quad$ if $\theta=0$ and $\mathbb{E}\left(X_{1}\right) \geq 0$, where

$$
-B<\operatorname{Re}\left(-\gamma_{p}(\theta)\right) \leq \cdots \leq \operatorname{Re}\left(-\gamma_{1}(\theta)\right)<-\gamma_{0}(\theta) \leq 0
$$

4. $-\gamma_{0}(\theta)$ is a simple (resp. double) zero of $\varphi_{\theta}$, if $\theta>0$, or $\theta=0$ and $\mathbb{E}\left(X_{1}\right) \neq 0$ (resp. otherwise, i.e. $\theta=0$ and $\left.\mathbb{E}\left(X_{1}\right)=0\right)$.

The zeros of $\varphi_{\theta}$ which are the poles of $\widehat{F}(\theta, \mu, \rho, \cdot)$ will play an important role in our approach, as shown below (see the proof of Th. 2.8 in Sect. 3.4, and properties in Sect. 4.5).

Theorem 2.8. Let us suppose $(H)$ and (2.23). Then for any $\theta \in[0, \kappa], \mu \geq 0$ and $\rho \geq 0$, there exists a positive number $C_{0}(\theta, \mu, \rho)>0$ and complex $x$-polynomial functions $C_{1}(\theta, \mu, \rho, x), \cdots, C_{p}(\theta, \mu, \rho, x)$ so that $F(\theta, \mu, \rho, x)$ has the following asymptotic expansion as $x \rightarrow \infty$ :

$$
\begin{aligned}
F(\theta, \mu, \rho, x)= & C_{0}(\theta, \mu, \rho) \mathrm{e}^{-\gamma_{0}(\theta) x}+\sum_{i=1}^{p} a_{i}\left(C_{i}(\theta, \mu, \rho, x) \mathrm{e}^{-\gamma_{i}(\theta) x}+\overline{C_{i}}(\theta, \mu, \rho, x) \mathrm{e}^{-\overline{\gamma_{i}}(\theta) x}\right) \\
& +\mathrm{O}\left(\mathrm{e}^{-B x}\right)
\end{aligned}
$$

where $a_{i}=\frac{1}{2}$ if $\gamma_{i}(\theta)$ is real and $a_{i}=1$ otherwise. The degree of $C_{i}(\theta, \mu, \rho,$.$) is n_{i}-1$, where $n_{i}$ is the order of multiplicity of $-\gamma_{i}(\theta)$. In addition, $\mathrm{O}$ is uniform with respect to $\mu \geq 0, \rho \geq 0$ and $\theta \in[0, \kappa]$.

\section{Remark 2.9.}

1. In (2.25), it is understood that $B$ may be chosen in $] 0, B_{\nu}\left[\right.$ closest as possible to $B_{\nu}$.

2. In Section 4.5 we study the coefficients $C_{i}(\theta, \mu, \rho, x)$.

3. Obviously, (2.25) and (2.24) imply that:

$$
\lim _{x \rightarrow \infty} F(\theta, \mu, \rho, x) \mathrm{e}^{\gamma_{0}(\theta) x}=C_{0}(\theta, \mu, \rho) .
$$

This property has already been reached in [2] in the particular case: $\theta=\mu=\rho=0$ :

$$
F(0,0,0, x)=\mathbb{P}\left(T_{x}<\infty\right) \sim C_{0}(0,0,0) \mathrm{e}^{-\gamma_{0}(0) x} \quad \text { as } \quad x \rightarrow \infty .
$$

(1) Heuristically, no assumption on the negative jumps is required to get:

$$
F(\theta, \mu, \rho, x) \leq C \mathrm{e}^{-\gamma_{0}(\theta) x}
$$


However to obtain an equivalent, or an asymptotic development of $F(\theta, \mu, \rho, x)$ when $x$ goes to infinity, it is natural to suppose that the negative and the positive parts of the jumps of $\left(X_{t}, t \geq 0\right)$ are controlled. Our asymptotic development looks like a perturbation theorem around the case of Brownian motion with negative drift.

\subsection{Polynomial decay}

According to item 3 of Remark 2.9, under $(H)$ and (2.23), the ruin probability goes to 0, with exponential rate. The aim is to prove that under weaker assumptions,

$$
F(x):=F(0,0,0, x)=\mathbb{P}\left(T_{x}<\infty\right)
$$

has a polynomial type rate of decay, as $x \rightarrow \infty$.

In this section we suppose neither $(H)$ nor $(2.23)$.

Theorem 2.10. Let us assume that

$$
\int_{\mathbb{R}}|y| \mathbb{1}_{\{|y|>1\}} \nu(\mathrm{d} y)<\infty \quad \text { and } \quad \mathbb{E}\left(X_{1}\right)=-c+\int_{\mathbb{R}} y \mathbb{1}_{\{|y| \geq 1\}} \nu(\mathrm{d} y)<0 .
$$

and

$$
\int_{0}^{\infty} y^{p} \nu(\mathrm{d} y)<\infty, \text { for some } \quad p \geq 2 .
$$

Let $n$ be the integer part of $p-2$, then

$$
\forall x \in \mathbb{R}_{+} \quad \mathbb{P}\left(T_{x}<\infty\right) \leq \frac{C_{n}}{1+x^{n}}
$$

where $C_{n}>0$.

\section{Proofs}

\subsection{Proof of Theorems 2.1 and 2.3}

Our approach is only based on the following estimate:

$$
F(\theta, \mu, \rho, x) \sim C_{0}(\theta, \mu, \rho) \mathrm{e}^{-\gamma_{0}(\theta) x} \quad \text { as } \quad x \rightarrow \infty
$$

where $F(\theta, \mu, \rho, x)$ (resp. $\left.\gamma_{0}(\theta)\right)$ is defined by (2.9) (resp. (1.25) and (1.26)). The coefficient $C_{0}(\theta, \mu, \rho)$ comes from (2.25) included in Theorem 2.8 .

Theorems 2.1 and 2.3 related to the three cases $\mathbb{E}\left(X_{1}\right)>0, \mathbb{E}\left(X_{1}\right)<0$ and $\mathbb{E}\left(X_{1}\right)=0$ may be proved by using the same technique. Therefore we will only consider the case $\mathbb{E}\left(X_{1}\right)=0$ and the case $\mathbb{E}\left(X_{1}\right)<0$ (see Sect. 4.6 too).

3.1 .1

Let us start with the case $\mathbb{E}\left(X_{1}\right)=0$.

(i) Since $\varphi\left(-\gamma_{0}(\theta)\right)=\theta, \varphi(0)=0$ and $\varphi^{\prime}(0)=0$, we may use the asymptotic expansion of $\varphi$ at 0 at order 2 :

$$
\theta=\varphi\left(-\gamma_{0}(\theta)\right)=\varphi(-h)=\frac{h^{2}}{2} \varphi^{\prime \prime}(0)+\mathrm{o}\left(h^{2}\right)
$$


Therefore:

$$
\gamma_{0}(\theta)=h=\sqrt{\frac{2 \theta}{\varphi^{\prime \prime}(0)}}+o(\sqrt{\theta}) .
$$

Recall that (3.1) is uniform with respect to $\theta \in[0, \kappa]$. Then replacing $\theta$ by $\frac{\theta}{x^{2}}$ in $(3.1)$ brings to:

$$
\begin{aligned}
F\left(\frac{\theta}{x^{2}}, \mu, \rho, x\right) & =\mathbb{E}\left(\mathrm{e}^{-\theta \frac{T_{x}}{x^{2}}-\mu K_{x}-\rho L_{x}} \mathbb{1}_{\left\{T_{x}<\infty\right\}}\right) \\
& \sim C_{0}\left(\frac{\theta}{x^{2}}, \mu, \rho\right) \mathrm{e}^{-\sqrt{\frac{2 \theta}{\varphi^{\prime \prime}(0)}}} \text { as } \quad x \rightarrow \infty \\
& \sim C_{0}(0, \mu, \rho) \mathrm{e}^{-\sqrt{\frac{2 \theta}{\varphi^{\prime \prime}(0)}}} \text { as } \quad x \rightarrow \infty
\end{aligned}
$$

(ii) We would like to demonstrate in this item that $C_{0}(0, \mu, \rho)$ and $\mathrm{e}^{-\sqrt{\frac{2 \theta}{\varphi^{\prime \prime}(0)}}}$ are Laplace transforms of probability measures on $\left[0, \infty\left[\times\left[0, \infty\left[\right.\right.\right.\right.$ and $\left[0, \infty\left[\right.\right.$ respectively. As for $\mathrm{e}^{-\sqrt{\frac{1}{\varphi^{\prime \prime}(0)}} \sqrt{2 \theta}}$, it is well known (cf. [12] p. 96 formula (8.6)) that it is the Laplace transform of the first hitting time to level $\sqrt{\frac{1}{\varphi^{\prime \prime}(0)}}$ for a standard Brownian motion started at 0 .

Let us prove that $C_{0}(0, \mu, \rho)$ is a Laplace transform. We modify the identity (4.34) in Section 4.5 via the relation:

Then, we obtain:

$$
\frac{\mathrm{e}^{-a y}-1+a y}{a^{2}}=-\int_{0}^{y}(z-y) \mathrm{e}^{-a z} \mathrm{~d} z .
$$

$$
\begin{aligned}
C_{0}(0, \mu, \rho)= & \frac{1}{\varphi^{\prime \prime}(0)}\left[1-2 \int_{0}^{\infty} \mathrm{e}^{-\rho y}\left(\int_{0}^{y}(z-y) \mathrm{e}^{(\rho-\mu) z} \mathrm{~d} z\right) \nu(\mathrm{d} y)\right. \\
& \left.-2 \int_{-\infty}^{0}\left(\int_{0}^{-y}(y+b) \mathbb{E}\left(\mathrm{e}^{-\mu K_{b}-\rho L_{b}} \mathbb{1}_{\left\{T_{b}<\infty\right\}}\right) \mathrm{d} b\right) \nu(\mathrm{d} y)\right] .
\end{aligned}
$$

Let $n(b, \mathrm{~d} u, \mathrm{~d} z)$ be the distribution of the couple $\left(K_{b}, L_{b}\right)$. Consequently, $C_{0}(0, \mu, \rho)$ may be written as follows:

$$
\begin{aligned}
C_{0}(0, \mu, \rho)= & \frac{1}{\varphi^{\prime \prime}(0)}\left[1+2 \int_{0}^{\infty} \mathrm{e}^{-\mu z}\left(\int_{[z, \infty[}(y-z) \mathrm{e}^{-\rho(y-z)} \nu(\mathrm{d} y)\right) \mathrm{d} z\right. \\
& \left.-2 \int_{0}^{\infty} \int_{0}^{\infty} \mathrm{e}^{-\mu u} \mathrm{e}^{-\rho z} \int_{-\infty}^{0}\left(\int_{0}^{-y}(y+b) n(b, \mathrm{~d} u, \mathrm{~d} z) \mathrm{d} b\right) \nu(\mathrm{d} y)\right] \\
= & \int_{0}^{\infty} \int_{0}^{\infty} \mathrm{e}^{-\mu u} \mathrm{e}^{-\rho z} w^{0}(\mathrm{~d} u, \mathrm{~d} z),
\end{aligned}
$$

with $w^{0}$ defined in $(2.6)$.

3.1 .2

Next we study the case $\mathbb{E}\left(X_{1}\right)<0$.

(i) On one hand, from (3.1), we have:

$$
\begin{aligned}
\mathbb{E}\left(\mathrm{e}^{-\theta T_{x}-\mu K_{x}-\rho L_{x}} \mid T_{x}<\infty\right) & =\frac{F(\theta, \mu, \rho, x)}{\mathbb{P}\left(T_{x}<\infty\right)}=\frac{F(\theta, \mu, \rho, x)}{F(0,0,0, x)} \\
& \sim \frac{C_{0}(\theta, \mu, \rho)}{C_{0}(0,0,0)} \mathrm{e}^{-\left(\gamma_{0}(\theta)-\gamma_{0}(0)\right) x} \quad \text { as } \quad x \rightarrow \infty
\end{aligned}
$$


On the other hand, the behavior of $\gamma_{0}(\theta)$ in a neighborhood of 0 is the following:

$$
\gamma_{0}(\theta)-\gamma_{0}(0)=-\frac{1}{\varphi^{\prime}\left(-\gamma_{0}(0)\right)} \theta+\frac{\varphi^{\prime \prime}\left(-\gamma_{0}(0)\right)}{2 \varphi^{\prime 3}\left(-\gamma_{0}(0)\right)} \theta^{2}+o\left(\theta^{2}\right) \quad(\theta \rightarrow 0) .
$$

Combining (3.7) and (3.8) leads after easy calculations to:

$$
\mathbb{E}\left(\mathrm{e}^{-\frac{\theta}{\sqrt{x}}\left(T_{x}+\frac{x}{\varphi^{\prime}\left(-\gamma_{0}(0)\right)}\right)-\mu K_{x}-\rho L_{x}} \mid T_{x}<\infty\right) \sim \frac{C_{0}(0, \mu, \rho)}{C_{0}(0,0,0)} \mathrm{e}^{-\frac{1}{2} \frac{\varphi^{\prime \prime}\left(-\gamma_{0}(0)\right)}{\varphi^{\prime 3}\left(-\gamma_{0}(0)\right)} \theta^{2}} \quad \text { as } \quad x \rightarrow \infty .
$$

Let us observe that $\mathrm{e}^{-\frac{1}{2} \frac{\varphi^{\prime \prime}\left(-\gamma_{0}(0)\right)}{\varphi^{\prime 3}\left(-\gamma_{0}(0)\right)}} \theta^{2}$ is the Laplace transform (with respect to the $\theta$ variable) of the Gaussian distribution with mean 0 and variance $\frac{\varphi^{\prime \prime}\left(-\gamma_{0}(0)\right)}{\varphi^{\prime 3}\left(-\gamma_{0}(0)\right)}$. As a result Theorem 2.1 will be proved as soon as we demonstrate that $\frac{C_{0}(0, \mu, \rho)}{C_{0}(0,0,0)}$ is the Laplace transform of a probability measure on $[0, \infty[\times[0, \infty[$. We proceed as in the case 3.1.1 above. We begin by using the relation (4.34):

$$
\begin{aligned}
\frac{C_{0}(\theta, \mu, \rho)}{C_{0}(0,0,0)}= & \frac{1}{C_{0}(0,0,0) \varphi^{\prime}\left(-\gamma_{0}(0)\right)}\left[-\frac{\gamma_{0}(0)}{2}+\int_{0}^{\infty} \frac{\mathrm{e}^{-\left(-\gamma_{0}(0)+\rho\right) y}-\mathrm{e}^{-\mu y}}{-\gamma_{0}(0)+\rho-\mu} \nu(\mathrm{d} y)\right. \\
& \left.-\int_{0}^{\infty} \frac{\mathrm{e}^{-\rho y}-\mathrm{e}^{-\mu y}}{\rho-\mu} \nu(\mathrm{d} y)+R F(0, \mu, \rho, .)\left(-\gamma_{0}(0)\right)\right] .
\end{aligned}
$$

It follows:

$$
\begin{aligned}
\frac{C_{0}(\theta, \mu, \rho)}{C_{0}(0,0,0)}= & \frac{1}{C_{0}(0,0,0) \varphi^{\prime}\left(-\gamma_{0}(0)\right)}\left[-\frac{\gamma_{0}(0)}{2}+\int_{0}^{\infty} \mathrm{e}^{-\mu y}\left(\int_{0}^{y}-\mathrm{e}^{-\left(-\gamma_{0}(0)+\rho-\mu\right) z} \mathrm{~d} z\right) \nu(\mathrm{d} y)\right. \\
& \left.-\int_{0}^{\infty} \mathrm{e}^{-\mu y}\left(\int_{0}^{y} \mathrm{e}^{-(\rho-\mu) z} \mathrm{~d} z\right) \nu(\mathrm{d} y)+R F(0, \mu, \rho, .)\left(-\gamma_{0}(0)\right)\right] .
\end{aligned}
$$

Using the Fubini theorem we get:

$$
\begin{aligned}
\frac{C_{0}(\theta, \mu, \rho)}{C_{0}(0,0,0)}= & \frac{1}{C_{0}(0,0,0) \varphi^{\prime}\left(-\gamma_{0}(0)\right)}\left[-\frac{\gamma_{0}(0)}{2}-\int_{0}^{\infty}\left(\mathrm{e}^{\gamma_{0}(0) z}-1\right) \mathrm{e}^{-\rho z}\left(\int_{[z ; \infty[} \mathrm{e}^{-\mu(y-z)} \nu(\mathrm{d} y)\right) \mathrm{d} z\right. \\
& \left.+\int_{-\infty}^{0}\left(\int_{0}^{-y}\left(\mathrm{e}^{\gamma_{0}(0) z}-1\right) \mathbb{E}\left(\mathrm{e}^{-\mu K_{b}-\rho L_{b}} \mathbb{1}_{\left\{T_{b}<\infty\right\}}\right) \mathrm{d} b\right) \nu(\mathrm{d} y)\right] .
\end{aligned}
$$

Let $n(b, \mathrm{~d} u, \mathrm{~d} z)$ be the distribution of $\left(K_{b}, L_{b}\right)$ conditionally on $\left\{T_{b}<\infty\right\}$. Then:

$$
\begin{aligned}
\frac{C_{0}(\theta, \mu, \rho)}{C_{0}(0,0,0)}= & \frac{1}{C_{0}(0,0,0) \varphi^{\prime}\left(-\gamma_{0}(0)\right)}\left[-\frac{\gamma_{0}(0)}{2}-\int_{0}^{\infty}\left(\mathrm{e}^{\gamma_{0}(0) z}-1\right) \mathrm{e}^{-\rho z}\left(\int_{[z ; \infty[} \mathrm{e}^{-\mu(y-z)} \nu(\mathrm{d} y)\right) \mathrm{d} z\right. \\
& \left.+\int_{0}^{\infty} \int_{0}^{\infty} \mathrm{e}^{-\mu u} \mathrm{e}^{-\rho z} \int_{-\infty}^{0}\left(\int_{0}^{-y}\left(\mathrm{e}^{\gamma_{0}(0) z}-1\right) \mathbb{P}\left(T_{b}<\infty\right) n(b, \mathrm{~d} u, \mathrm{~d} z) \mathrm{d} b\right) \nu(\mathrm{d} y)\right] \\
& =\int_{0}^{\infty} \int_{0}^{\infty} \mathrm{e}^{-\mu u} \mathrm{e}^{-\rho z} w^{-}(\mathrm{d} u, \mathrm{~d} z),
\end{aligned}
$$

with $w^{-}$defined in (2.1). 


\subsection{Proof of Theorem 2.4}

In this subsection it is assumed that: $\lambda:=\nu(\mathbb{R})<\infty$. Then $\left(J_{t}, t \geq 0\right)$ is a compound Poisson process. As a result, it admits a first jump time $\tau_{1}$, exponentially distributed with parameter $\nu(\mathbb{R})(c f$. [18], Th. 21.1). The process $\left(X_{t+\tau_{1}}-X_{\tau_{1}} ; t \geq 0\right)$ is again a Lévy process distributed as $\left(X_{t}, t \geq 0\right)$. This property is the key of our approach that we will briefly describe. Let us consider three cases:

- $T_{x}=\inf \left\{t \geq 0 ; B_{t}-c_{0} t>x\right\}<\tau_{1} \quad$ if $\sup _{0 \leq t \leq \tau_{1}}\left(B_{t}-c_{0} t\right)>x$,

- $T_{x}=\tau_{1} \quad$ if $\quad \sup _{0 \leq t \leq \tau_{1}}\left(B_{t}-c_{0} t\right)<x \quad$ and $\quad J_{\tau_{1}}+B_{\tau_{1}}-c_{0} \tau_{1}>x$,

- $T_{x}>\tau_{1}$ otherwise. Then, conditionally on $\left\{T_{x}>\tau_{1}\right\}, T_{x}-\tau_{1}$ is distributed as $\widehat{T}_{x-X_{\tau_{1}}}$ where $\left(\widehat{T}_{x} ; x>0\right)$ is an independent copy of $\left(T_{x} ; x>0\right)$. $\left(\widehat{T}_{x} ; x>0\right)$ is independent from $\left(X_{t}, t \geq 0\right)$ as well. This "renewal" part gives rise to the integral kernel $\Lambda_{\theta}$ defined in (2.14).

This leads us to decompose $F(\theta, \mu, \rho,$.$) defined by (2.9)$, as follows:

$$
\begin{aligned}
F(\theta, \mu, \rho, x)= & \mathbb{E}\left(\mathrm{e}^{-\theta T_{x}-\mu K_{x}-\rho L_{x}} \mathbb{1}_{\left\{T_{x}<\tau_{1}\right\}}\right)+\mathbb{E}\left(\mathrm{e}^{-\theta T_{x}-\mu K_{x}-\rho L_{x}} \mathbb{1}_{\left\{T_{x}=\tau_{1}\right\}}\right) \\
& +\mathbb{E}\left(\mathrm{e}^{-\theta T_{x}-\mu K_{x}-\rho L_{x}} \mathbb{1}_{\left\{\tau_{1}<T_{x}<\infty\right\}}\right) .
\end{aligned}
$$

We will calculate the two first terms of (3.14) in Lemmas 3.1, 3.2. The third one will be determined in Lemma 3.3.

Lemma 3.1. Let $\alpha_{\theta}$ be the real number, defined in (2.11), then:

$$
\mathbb{E}\left(\mathrm{e}^{-\theta T_{x}-\mu K_{x}-\rho L_{x}} \mathbb{1}_{\left\{T_{x}<\tau_{1}\right\}}\right)=\mathrm{e}^{-\left(c_{0}+\alpha_{\theta}\right) x} .
$$

Proof of Lemma 3.1. Let $\left(\widetilde{B}_{t}, t \geq 0\right)$ be the Brownian motion with drift $-c_{0}$ :

$$
\widetilde{B}_{t}:=B_{t}-c_{0} t \quad \forall t \geq 0 .
$$

We set $\widetilde{T}_{x}:=\inf \left\{t \geq 0 ; \widetilde{B}_{t}>x\right\}, x \geq 0$.

Then, $\left\{T_{x}<\tau_{1}\right\}=\left\{\widetilde{T}_{x}<\tau_{1}\right\}$ a.s. and $K_{x}=L_{x}=0$ on $\left\{T_{x}<\tau_{1}\right\}$.

Since $\tau_{1}$ is exponentially distributed with parameter $\lambda$ and is independent from $\widetilde{T}_{x}$, we have:

$$
\mathbb{E}\left(\mathrm{e}^{-\theta T_{x}-\mu K_{x}-\rho L_{x}} \mathbb{1}_{\left\{T_{x}<\tau_{1}\right\}}\right)=\mathbb{E}\left(\mathrm{e}^{-(\lambda+\theta) \widetilde{T}_{x}}\right)
$$

According to ([12], exercise 5.10 p. 197) we can conclude that (3.15) holds.

Lemma 3.2. We have:

$$
\begin{aligned}
\mathbb{E}\left(\mathrm{e}^{-\theta T_{x}-\mu K_{x}-\rho L_{x}} \mathbb{1}_{\left\{T_{x}=\tau_{1}\right\}}\right)=\frac{\mathrm{e}^{-\left(c_{0}+\alpha_{\theta}\right) x}}{\alpha_{\theta}\left(\mu-\rho+c_{0}+\alpha_{\theta}\right)} \int_{[0, x]}\left(\mathrm{e}^{\left(-\rho+c_{0}+\alpha_{\theta}\right) y}-\mathrm{e}^{-\mu y}\right) \nu(\mathrm{d} y) \\
+\frac{\mathrm{e}^{-\rho x}}{\alpha_{\theta}\left(\mu-\rho+c_{0}-\alpha_{\theta}\right)} \int_{] x, \infty[}\left(\mathrm{e}^{-\left(\rho+\alpha_{\theta}-c_{0}\right)(y-x)}-\mathrm{e}^{-\mu(y-x)}\right) \nu(\mathrm{d} y) \\
+\frac{\mathrm{e}^{(\mu-\rho) x}-\mathrm{e}^{-\left(c_{0}+\alpha_{\theta}\right) x}}{\alpha_{\theta}\left(\mu-\rho+c_{0}+\alpha_{\theta}\right)} \int_{] x, \infty[} \mathrm{e}^{-\mu y} \nu(\mathrm{d} y) \\
\quad-\frac{\mathrm{e}^{-\left(c_{0}+\alpha_{\theta}\right) x}}{\alpha_{\theta}\left(\mu-\rho+c_{0}-\alpha_{\theta}\right)} \int_{0}^{\infty}\left(\mathrm{e}^{-\left(\rho+\alpha_{\theta}-c_{0}\right) y}-\mathrm{e}^{-\mu y}\right) \nu(\mathrm{d} y) .
\end{aligned}
$$


Proof of Lemma 3.2. Let us write: $Y_{1}:=J_{\tau_{1}}$. We observe that on $\left\{T_{x}=\tau_{1}\right\}, Y_{1}>0$. Morever:

$$
\left\{T_{x}=\tau_{1}\right\}=\left\{\sup _{t \leq \tau_{1}} \widetilde{B}_{t}<x, \widetilde{B}_{\tau_{1}}+Y_{1}>x\right\}
$$

and

$$
K_{x}=\widetilde{B}_{\tau_{1}}+Y_{1}-x \quad, \quad L_{x}=x-\widetilde{B}_{\tau_{1}},
$$

where $\left(\widetilde{B}_{t}, t \geq 0\right)$ is defined by the relation (3.16). Conditioning by $\left(\tau_{1}, Y_{1}\right)$ implies that:

$$
\begin{aligned}
\Delta & :=\mathbb{E}\left(\mathrm{e}^{-\theta T_{x}-\mu K_{x}-\rho L_{x}} \mathbb{1}_{\left\{T_{x}=\tau_{1}\right\}}\right) \\
& =\mathrm{e}^{-\rho x} \int_{0}^{\infty} \mathrm{d} t \mathrm{e}^{-(\lambda+\theta) t} \int_{0}^{\infty} \nu(\mathrm{d} y) \mathbb{E}\left(\mathrm{e}^{-(\mu-\rho) \widetilde{B}_{t}-\mu(y-x)} \mathbb{1}_{\left\{\sup _{u \leq t} \widetilde{B}_{u}<x ; x-y \leq \widetilde{B}_{t}\right\}}\right)
\end{aligned}
$$

since the distribution of $Y_{1}$ is $\frac{1}{\lambda} \nu$.

The density function of $\left(\sup _{u \leq t} B_{u}, B_{t}\right)$ is given in ([12] p. 95), i.e.:

$$
\mathbb{P}\left(B_{t} \in \mathrm{d} a ; \sup _{u \leq t} B_{u} \in \mathrm{d} b\right)=\frac{2(2 b-a)}{\sqrt{2 \pi t^{3}}} \mathrm{e}^{-\frac{(2 b-a)^{2}}{2 t}} \mathbb{1}_{\{a<b ; b>0\}} \mathrm{d} a \mathrm{~d} b .
$$

Let us apply Girsanov's formula:

$$
\mathbb{P}\left(\widetilde{B}_{t} \in d a ; \sup _{u \leq t} \widetilde{B}_{u} \in \mathrm{d} b\right)=\frac{2(2 b-a)}{\sqrt{2 \pi t^{3}}} \mathrm{e}^{-c_{0} a-\frac{c_{0}^{2}}{2} t} \mathrm{e}^{-\frac{(2 b-a)^{2}}{2 t}} \mathbb{1}_{\{a<b ; b>0\}} \mathrm{d} a \mathrm{~d} b
$$

Combining (3.23) and (3.21) leads to:

$$
\begin{aligned}
\Delta=\mathrm{e}^{(\mu-\rho) x} \int_{0}^{\infty} \nu(\mathrm{d} y) \mathrm{e}^{-\mu y} & \int_{x-y}^{x} \mathrm{~d} a \mathrm{e}^{-\left(c_{0}+\mu-\rho\right) a} \int_{a \vee 0}^{x} \mathrm{~d} b(2 b-a) \\
& \int_{0}^{\infty} \frac{2}{\sqrt{2 \pi t^{3}}} \mathrm{e}^{-\frac{1}{2}\left(\left(2(\lambda+\theta)+c_{0}^{2}\right) t+\frac{(2 b-a)^{2}}{t}\right)} \mathrm{d} t .
\end{aligned}
$$

Let us recall the classical identities (cf. [14] p. 118, or [9] Sects. 8.4326 p. 959, and 8.4693 p. 967):

$$
\mathcal{K}_{\frac{1}{2}}(\delta):=\frac{1}{2} \int_{0}^{\infty} \frac{1}{\sqrt{t}} \mathrm{e}^{-\frac{\delta}{2}\left(t+\frac{1}{t}\right)} \mathrm{d} t=\sqrt{\frac{\pi}{2 \delta}} \mathrm{e}^{-\delta} \quad \forall \delta>0,
$$

and

$$
\int_{0}^{\infty} \frac{1}{\sqrt{t^{3}}} \mathrm{e}^{-\frac{1}{2}\left(\beta t+\frac{\gamma}{t}\right)} \mathrm{d} t=\sqrt{\frac{2 \pi}{\gamma}} \mathrm{e}^{-\sqrt{\beta \gamma}} \quad \forall \beta>0, \forall \gamma>0,
$$

obtained by derivation and the changing of variable $t \rightarrow \sqrt{\frac{\beta}{\gamma}} t$.

This allows to first compute explicitly the integral with respect to $\mathrm{d} t$ in (3.24):

$$
\Delta=2 \mathrm{e}^{(\mu-\rho) x} \int_{0}^{\infty} \nu(\mathrm{d} y) \mathrm{e}^{-\mu y} \int_{x-y}^{x} \mathrm{~d} a \mathrm{e}^{-\left(c_{0}+\mu-\rho\right) a} \int_{a \vee 0}^{x} \mathrm{e}^{-\alpha_{\theta}(2 b-a)} \mathrm{d} b .
$$


In a second step we evaluate the integral with respect to $\mathrm{d} b$ :

$$
\Delta=\frac{\mathrm{e}^{(\mu-\rho) x}}{\alpha_{\theta}} \int_{0}^{\infty} \nu(\mathrm{d} y) \mathrm{e}^{-\mu y} \int_{x-y}^{x} \mathrm{~d} a \mathrm{e}^{-\left(c_{0}+\mu-\rho\right) a}\left(\mathrm{e}^{-\alpha_{\theta}|a|}-\mathrm{e}^{-\alpha_{\theta}(2 x-a)}\right)
$$

Let us introduce two cases $x-y \geq 0$ and $x-y<0$ :

$$
\begin{aligned}
\Delta= & \frac{\mathrm{e}^{(\mu-\rho) x}}{\alpha_{\theta}} \int_{[0, x]} \nu(\mathrm{d} y) \mathrm{e}^{-\mu y} \int_{x-y}^{x} \mathrm{e}^{-\left(c_{0}+\mu-\rho+\alpha_{\theta}\right) a} \mathrm{~d} a \\
& +\frac{\mathrm{e}^{(\mu-\rho) x}}{\alpha_{\theta}} \int_{] x, \infty[} \nu(\mathrm{d} y) \mathrm{e}^{-\mu y}\left[\int_{x-y}^{0} \mathrm{e}^{-\left(c_{0}+\mu-\rho-\alpha_{\theta}\right) a} \mathrm{~d} a+\int_{0}^{x} \mathrm{e}^{-\left(c_{0}+\mu-\rho+\alpha_{\theta}\right) a} \mathrm{~d} a\right] \\
& -\frac{\mathrm{e}^{\left(-2 \alpha_{\theta}+\mu-\rho\right) x}}{\alpha_{\theta}} \int_{0}^{\infty} \nu(\mathrm{d} y) \mathrm{e}^{-\mu y} \int_{x-y}^{x} \mathrm{e}^{-\left(c_{0}+\mu-\rho-\alpha_{\theta}\right) a} \mathrm{~d} a .
\end{aligned}
$$

If the integral is computed according to $\mathrm{d} a$, we may easily obtain (3.18).

Lemma 3.3. In (3.14), the third expectation is equal to:

$$
\mathbb{E}\left(\mathrm{e}^{-\theta T_{x}-\mu K_{x}-\rho L_{x}} \mathbb{1}_{\left\{\tau_{1}<T_{x}<\infty\right\}}\right)=\frac{1}{\alpha_{\theta}} \int_{-\infty}^{\infty} \nu(\mathrm{d} y) \int_{-\infty}^{(x-y) \wedge x} \mathrm{e}^{-c_{0} a}\left(\mathrm{e}^{-\alpha_{\theta}|a|}-\mathrm{e}^{(2 x-a) \alpha_{\theta}}\right) F(\theta, \mu, \rho, x-a-y) \mathrm{d} a
$$

Morever:

$$
\mathrm{e}^{-\alpha_{\theta}|a|}-\mathrm{e}^{-(2 x-a) \alpha_{\theta}} \geq 0 \quad \text { if } \quad a \leq(x-y) \wedge x,
$$

so $\Lambda_{\theta}$ defined by (2.14) is an non-negative operator.

Proof of Lemma 3.3. Formula (3.30) may be proved proceeding analogously to the proof of previous lemma.

Remark 3.4. By introducing adapted functional Banach spaces, we may study in Section 4.3 (cf. Th. 4.2 and Prop. 4.3) the operator $\Lambda_{\theta}$. We can prove that $F(\theta, \mu, \rho,$.$) is the unique solution of (2.10). Moreover, if \theta>0$, or $\theta=0$ and $\mathbb{E}\left(X_{1}\right)<0$, then $F(\theta, \mu, \rho,$.$) has a sub-exponential rate of decay:$

$$
F(\theta, \mu, \rho, x) \leq C \mathrm{e}^{-\gamma x}, \quad \forall x \geq 0, \quad \text { for some } \quad C>0, \gamma>0
$$

The optimal value of $\gamma$ has be given in Remark 2.9.

Note that if $\theta=0$ and $\mathbb{E}\left(X_{1}\right) \geq 0$, then $F(0,0,0, x)=1$, hence there is no hope to obtain a sub-exponential rate of decay.

\subsection{Proof of Theorem 2.5}

Assume that $\nu$ satisfies the assumptions given in Theorem 2.5. The proof of Theorem 2.5 will be divided into two steps. We will first prove (2.17) when $\nu(\mathbb{R})<\infty$. In a second step, we will approximate $\nu$ by a sequence of finite measures $\left(\nu_{n}\right)$. Then by replacing $\nu$ by $\nu_{n}$, and by taking the limit $n \rightarrow \infty$, it will be proved that (2.17) remains valid. 
Step 1. Let us suppose that $\nu(\mathbb{R})<\infty$.

a) Taking the Laplace transform in functional equation (2.10) leads to:

$$
\widehat{F}(\theta, \mu, \rho, q)=\widehat{F_{0}}(\theta, \mu, \rho, q)+\widehat{F_{1}}(\theta, \mu, \rho, q)+\widehat{\Lambda_{\theta} F}(\theta, \mu, \rho, .)(q) \quad q \in D_{0}
$$

with $D_{0}:=\{z \in \mathbb{C}, \operatorname{Re}(z)>0\}$.

The identity (2.12) implies:

$$
\widehat{F_{0}}(\theta, \mu, \rho, q)=\frac{1}{c+\alpha_{\theta}+q} .
$$

As for $\widehat{F}_{1}(\theta, \mu, \rho, x)$, starting from $(2.13)$, we split the integral in four parts:

$$
\begin{aligned}
\widehat{F_{1}}(\theta, \mu, \rho, q) & =\int_{0}^{\infty} \mathrm{e}^{-q x} F_{1}(\theta, \mu, \rho, x) \mathrm{d} x \\
& =I_{1}(\theta, \mu, \rho, q)+I_{2}(\theta, \mu, \rho, q)+I_{3}(\theta, \mu, \rho, q)+I_{4}(\theta, \mu, \rho, q)
\end{aligned}
$$

where

$$
\begin{aligned}
& I_{1}(\theta, \mu, \rho, q):=\int_{0}^{\infty} \frac{\mathrm{e}^{-\left(q+c+\alpha_{\theta}\right) x}}{\alpha_{\theta}\left(\mu+c+\alpha_{\theta}-\rho\right)}\left(\int_{[0, x]}\left(\mathrm{e}^{\left(c+\alpha_{\theta}-\rho\right) y}-\mathrm{e}^{-\mu y}\right) \nu(\mathrm{d} y)\right) \mathrm{d} x \\
& =\frac{1}{\alpha_{\theta}\left(q+c+\alpha_{\theta}\right)\left(\mu+c+\alpha_{\theta}-\rho\right)} \int_{0}^{\infty}\left(\mathrm{e}^{-(q+\rho) y}-\mathrm{e}^{-\left(q+\mu+c+\alpha_{\theta}\right) y}\right) \nu(\mathrm{d} y), \\
& I_{2}(\theta, \mu, \rho, q):=\int_{0}^{\infty} \frac{\mathrm{e}^{-(q+\rho) x}}{\alpha_{\theta}\left(\mu+c-\alpha_{\theta}-\rho\right)}\left(\int_{] x, \infty[}\left(\mathrm{e}^{-\left(\rho+\alpha_{\theta}-c\right)(y-x)}-\mathrm{e}^{-\mu(y-x)}\right) \nu(\mathrm{d} y)\right) \mathrm{d} x \\
& =\frac{1}{\alpha_{\theta}\left(\mu+c-\alpha_{\theta}-\rho\right)} \int_{0}^{\infty}\left(\frac{\mathrm{e}^{-\left(\rho+\alpha_{\theta}-c\right) y}-\mathrm{e}^{-(q+\rho) y}}{q+c-\alpha_{\theta}}+\frac{\mathrm{e}^{-(q+\rho) y}-\mathrm{e}^{-\mu y}}{q+\rho-\mu}\right) \nu(\mathrm{d} y), \\
& I_{3}(\theta, \mu, \rho, q):=\int_{0}^{\infty} \frac{\mathrm{e}^{-(q+\rho-\mu) x}-\mathrm{e}^{-\left(q+c+\alpha_{\theta}\right) x}}{\alpha_{\theta}\left(\mu-\rho+c+\alpha_{\theta}\right)}\left(\int_{] x, \infty[} \mathrm{e}^{-\mu y} \nu(\mathrm{d} y)\right) \mathrm{d} x \\
& =-\frac{1}{\alpha_{\theta}\left(\mu-\rho+c+\alpha_{\theta}\right)} \int_{0}^{\infty} \nu(\mathrm{d} y)\left(\frac{\mathrm{e}^{-(q+\rho) y}-\mathrm{e}^{-\mu y}}{q+\rho-\mu}-\frac{\mathrm{e}^{-\left(q+\mu+c+\alpha_{\theta}\right) y}-\mathrm{e}^{-\mu y}}{q+c+\alpha_{\theta}}\right), \\
& I_{4}(\theta, \mu, \rho, q):=-\int_{0}^{\infty} \frac{\mathrm{e}^{-\left(q+c+\alpha_{\theta}\right) x}}{\alpha_{\theta}\left(-\rho+\mu+c-\alpha_{\theta}\right)} \mathrm{d} x\left(\widehat{\nu^{+}}\left(\rho+\alpha_{\theta}-c\right)-\widehat{\nu^{+}}(\mu)\right) \\
& =\frac{\widehat{\nu^{+}}(\mu)-\widehat{\nu^{+}}\left(\rho+\alpha_{\theta}-c\right)}{\alpha_{\theta}\left(q+c+\alpha_{\theta}\right)\left(-\rho+\mu+c-\alpha_{\theta}\right)} \text {, }
\end{aligned}
$$

and $\widehat{\nu^{+}}$denotes the Laplace transform of $\nu_{\left.\right|_{\mathrm{j} 0, \infty]}}$ :

$$
\left.\widehat{\nu^{+}}(q):=\widehat{\nu}_{\mid \mathrm{j}, \infty]}(q)=\int_{0}^{\infty} \mathrm{e}^{-q y} \nu(\mathrm{d} y), \quad q \in\right]-r_{\nu}, \infty[
$$


Therefore:

$$
\begin{aligned}
\widehat{F_{1}}(\theta, \mu, \rho, q) & =-\frac{\widehat{\nu^{+}}(q+\rho)}{\alpha_{\theta}\left(q+c+\alpha_{\theta}\right)(q+\rho-\mu)}+\frac{\widehat{\nu^{+}}(q+\rho)}{\alpha_{\theta}\left(q+c-\alpha_{\theta}\right)(q+\rho-\mu)} \\
& +\frac{2 \widehat{\nu^{+}}\left(\rho+\alpha_{\theta}-c\right)}{\left(\mu+c-\alpha_{\theta}-\rho\right)\left(q+c-\alpha_{\theta}\right)\left(q+c+\alpha_{\theta}\right)} \\
& -\frac{2 \widehat{\nu^{+}}(\mu)}{\left(\mu+c-\alpha_{\theta}-\rho\right)(q+\rho-\mu)\left(q+c+\alpha_{\theta}\right)} .
\end{aligned}
$$

Let us introduce:

$$
C_{\theta}(q):=\left(q+c+\alpha_{\theta}\right)\left(q+c-\alpha_{\theta}\right)=q^{2}+2 c q-2(\lambda+\theta) .
$$

A straight calculation gives:

$$
\widehat{F_{1}}(\theta, \mu, \rho, q)=\frac{2}{C_{\theta}(q)}\left(\frac{\widehat{\nu^{+}}(q+\rho)-\widehat{\nu^{+}}(\mu)}{q+\rho-\mu}-\frac{\widehat{\nu^{+}}(\mu)-\widehat{\nu^{+}}\left(\rho+\alpha_{\theta}-c\right)}{\mu-\rho+c-\alpha_{\theta}}\right) .
$$

b) Let us now compute $\widehat{\Lambda_{\theta} F}(\theta, \mu, \rho,).(q)$.

Setting $b=x-a-y$ in (2.14) leads to:

$$
\begin{aligned}
& \Lambda_{\theta} F(\theta, \mu, \rho, .)(x) \\
& =\frac{1}{\alpha_{\theta}} \int_{-\infty}^{\infty} \nu(\mathrm{d} y) \int_{0}^{\infty} \mathrm{e}^{-c(x-b-y)}\left(\mathrm{e}^{-\alpha_{\theta}|x-y-b|}-\mathrm{e}^{-(x+y+b) \alpha_{\theta}}\right) F(\theta, \mu, \rho, b) \mathrm{d} b \\
& \quad-\frac{1}{\alpha_{\theta}} \int_{-\infty}^{0} \nu(\mathrm{d} y) \int_{0}^{-y} \mathrm{e}^{-c(x-b-y)}\left(\mathrm{e}^{-\alpha_{\theta}(x-y-b)}-\mathrm{e}^{-(x+y+b) \alpha_{\theta}}\right) F(\theta, \mu, \rho, b) \mathrm{d} b \\
& =H_{1} F(\theta, \mu, \rho, .)(x)+I(\theta, \mu, \rho, x) \\
& \quad+\frac{\mathrm{e}^{-\left(c+\alpha_{\theta}\right) x}}{\alpha_{\theta}}\left(R F(\theta, \mu, \rho, .)\left(\alpha_{\theta}-c\right)-R F(\theta, \mu, \rho, .)\left(-\alpha_{\theta}-c\right)\right),
\end{aligned}
$$

where we recall that $R$ has been defined by (2.16), and

$$
\begin{gathered}
H_{1} F(\theta, \mu, \rho, .)(x):=\frac{1}{\alpha_{\theta}} \int_{-\infty}^{\infty} \nu(\mathrm{d} y) \int_{0}^{\infty} \mathrm{e}^{-c(x-b-y)} \mathrm{e}^{-\alpha_{\theta}|x-y-b|} F(\theta, \mu, \rho, b) \mathrm{d} b, \\
I(\theta, \mu, \rho, x):=-\frac{1}{\alpha_{\theta}} \int_{-\infty}^{\infty} \nu(\mathrm{d} y) \int_{0}^{\infty} \mathrm{e}^{-c(x-b-y)} \mathrm{e}^{-(x+y+b) \alpha_{\theta}} F(\theta, \mu, \rho, b) \mathrm{d} b \\
=\frac{1}{\alpha_{\theta}} \mathrm{e}^{-\left(c+\alpha_{\theta}\right) x} \widehat{\nu}\left(\alpha_{\theta}-c\right) \widehat{F}\left(\theta, \mu, \rho, \alpha_{\theta}-c\right),
\end{gathered}
$$

and $\widehat{\nu}$ stands for the Laplace transform of $\nu$ :

$$
\left.\widehat{\nu}(q):=\int_{-\infty}^{\infty} \mathrm{e}^{-q y} \nu(\mathrm{d} y), \quad q \in\right]-r_{\nu}, \infty[
$$


We multiply both sides of (3.44) by $\mathrm{e}^{-q x}$ and we integrate with respect to the Lebesgue measure restricted to $[0, \infty[$. We get:

$$
\begin{aligned}
\widehat{\Lambda_{\theta} F}(\theta, \mu, \rho, .)(q)= & \widehat{H_{1} F}(\theta, \mu, \rho, .)(q)-\frac{\widehat{\nu}\left(\alpha_{\theta}-c\right) \widehat{F}\left(\theta, \mu, \rho, \alpha_{\theta}-c\right)}{\alpha_{\theta}\left(q+c+\alpha_{\theta}\right)} \\
& +\frac{R F(\theta, \mu, \rho, .)\left(\alpha_{\theta}-c\right)-R F(\theta, \mu, \rho, .)\left(-\alpha_{\theta}-c\right)}{\alpha_{\theta}\left(q+c+\alpha_{\theta}\right)}
\end{aligned}
$$

We now focus on the calculation of $\widehat{H_{1} F}(\theta, \mu, \rho,).(q)$. By using $(3.45)$, we come to:

$$
\begin{aligned}
\widehat{H_{1} F}(\theta, \mu, \rho, .)(q)= & \frac{1}{\alpha_{\theta}} \int_{-\infty}^{\infty} \mathrm{e}^{c y} \nu(\mathrm{d} y) \int_{0}^{\infty} \mathrm{e}^{c b} F(\theta, \mu, \rho, b) \mathrm{d} b \int_{0}^{\infty} \mathrm{e}^{-(q+c) x} \mathrm{e}^{-\alpha_{\theta}|x-y-b|} \mathrm{d} x \\
= & \frac{1}{\alpha_{\theta}} \int_{-\infty}^{0} \mathrm{e}^{c y} \nu(\mathrm{d} y) \int_{0}^{-y} \mathrm{e}^{c b} F(\theta, \mu, \rho, b) \mathrm{d} b \int_{0}^{\infty} \mathrm{e}^{-(q+c) x} \mathrm{e}^{-\alpha_{\theta}(x-y-b)} \mathrm{d} x \\
& +\frac{1}{\alpha_{\theta}} \int_{-\infty}^{\infty} \mathrm{e}^{c y} \nu(\mathrm{d} y) \int_{0 \vee(-y)}^{\infty} \mathrm{e}^{c b} F(\theta, \mu, \rho, b) \mathrm{d} b \int_{0}^{b+y} \mathrm{e}^{-(q+c) x} \mathrm{e}^{\alpha_{\theta}(x-y-b)} \mathrm{d} x \\
& +\frac{1}{\alpha_{\theta}} \int_{-\infty}^{\infty} \mathrm{e}^{c y} \nu(\mathrm{d} y) \int_{0 \vee(-y)}^{\infty} \mathrm{e}^{c b} F(\theta, \mu, \rho, b) \mathrm{d} b \int_{b+y}^{\infty} \mathrm{e}^{-(q+c) x} \mathrm{e}^{-\alpha_{\theta}(x-y-b)} \mathrm{d} x
\end{aligned}
$$

The $x$-integrals can be computed:

$$
\begin{aligned}
\widehat{H_{1} F}(\theta, \mu, \rho, .)(q)= & \frac{1}{\alpha_{\theta}} \int_{-\infty}^{0} \mathrm{e}^{\left(c+\alpha_{\theta}\right) y} \nu(\mathrm{d} y) \int_{0}^{-y} \mathrm{e}^{\left(c+\alpha_{\theta}\right) b} F(\theta, \mu, \rho, b) \mathrm{d} b \frac{1}{q+c+\alpha_{\theta}} \\
& +\frac{1}{\alpha_{\theta}} \int_{-\infty}^{\infty} \mathrm{e}^{\left(c-\alpha_{\theta}\right) y} \nu(\mathrm{d} y) \int_{0 \vee(-y)}^{\infty} \mathrm{e}^{\left(c-\alpha_{\theta}\right) b} F(\theta, \mu, \rho, b) \frac{1-\mathrm{e}^{-\left(q+c-\alpha_{\theta}\right)(b+y)}}{q+c-\alpha_{\theta}} \mathrm{d} b \\
& +\frac{1}{\alpha_{\theta}} \int_{-\infty}^{\infty} \mathrm{e}^{\left(c+\alpha_{\theta}\right) y} \nu(\mathrm{d} y) \int_{0 \vee(-y)}^{\infty} \mathrm{e}^{\left(c+\alpha_{\theta}\right) b} F(\theta, \mu, \rho, b) \frac{\mathrm{e}^{-\left(q+c+\alpha_{\theta}\right)(b+y)}}{q+c+\alpha_{\theta}} \mathrm{d} b .
\end{aligned}
$$

By using (2.16) we may obtain:

$$
\begin{aligned}
\widehat{H_{1} F}(\theta, \mu, \rho, .)(q)= & \frac{1}{\alpha_{\theta}\left(q+c+\alpha_{\theta}\right)}\left[R F(\theta, \mu, \rho, .)\left(-\alpha_{\theta}-c\right)+\int_{-\infty}^{0} \nu(\mathrm{d} y) \int_{0}^{-y} F(\theta, \mu, \rho, b) \mathrm{d} b\right] \\
& +\frac{1}{\alpha_{\theta}\left(q+c-\alpha_{\theta}\right)} \int_{-\infty}^{\infty} \mathrm{e}^{\left(c-\alpha_{\theta}\right) y} \nu(\mathrm{d} y) \int_{0 \vee(-y)}^{\infty} \mathrm{e}^{\left(c-\alpha_{\theta}\right) b} F(\theta, \mu, \rho, b) \mathrm{d} b \\
& -\frac{1}{\alpha_{\theta}\left(q+c-\alpha_{\theta}\right)} \int_{-\infty}^{\infty} \mathrm{e}^{-q y} \nu(\mathrm{d} y) \int_{0 \vee(-y)}^{\infty} \mathrm{e}^{-q b} F(\theta, \mu, \rho, b) \mathrm{d} b \\
& +\frac{1}{\alpha_{\theta}\left(q+c+\alpha_{\theta}\right)} \int_{-\infty}^{\infty} \mathrm{e}^{-q y} \nu(\mathrm{d} y) \int_{0 \vee(-y)}^{\infty} \mathrm{e}^{-q b} F(\theta, \mu, \rho, b) \mathrm{d} b .
\end{aligned}
$$

Since

$$
\frac{1}{\alpha_{\theta}\left(q+c+\alpha_{\theta}\right)}-\frac{1}{\alpha_{\theta}\left(q+c-\alpha_{\theta}\right)}=-\frac{2}{C_{\theta}(q)}
$$


we get:

$$
\begin{aligned}
\widehat{H_{1} F}(\theta, \mu, \rho, .)(q)= & \frac{R F(\theta, \mu, \rho, .)\left(-\alpha_{\theta}-c\right)}{\alpha_{\theta}\left(q+c+\alpha_{\theta}\right)} \\
& +\frac{\widehat{\nu}\left(\alpha_{\theta}-c\right) \widehat{F}\left(\theta, \mu, \rho, \alpha_{\theta}-c\right)-R F(\theta, \mu, \rho, .)\left(\alpha_{\theta}-c\right)}{\alpha_{\theta}\left(q+c-\alpha_{\theta}\right)} \\
& -\frac{2}{C_{\theta}(q)}(\widehat{\nu}(q) \widehat{F}(\theta, \mu, \rho, q)-R F(\theta, \mu, \rho, .)(q)) .
\end{aligned}
$$

Combining (3.33) and (3.48) gives:

$$
\begin{gathered}
\widehat{F}(\theta, \mu, \rho, q)\left(1+\frac{2 \widehat{\nu}(q)}{C_{\theta}(q)}\right)=\widehat{F}_{0}(\theta, \mu, \rho, q)+\widehat{F}_{1}(\theta, \mu, \rho, q)+\frac{2}{C_{\theta}(q)} R F(\theta, \mu, \rho, .)(q) \\
+\frac{2}{C_{\theta}(q)}\left(\widehat{\nu}\left(\alpha_{\theta}-c\right) \widehat{F}\left(\theta, \mu, \rho, \alpha_{\theta}-c\right)-R F(\theta, \mu, \rho, .)\left(\alpha_{\theta}-c\right)\right)
\end{gathered}
$$

As $C_{\theta}(q)+2 \widehat{\nu}(q)=2(\varphi(q)-\theta)$, it is easy to check:

$$
\begin{aligned}
(\varphi(q)-\theta) \widehat{F}(\theta, \mu, \rho, q)= & \frac{1}{2} C_{\theta}(q)\left[\widehat{F}_{0}(\theta, \mu, \rho, q)+\widehat{F}_{1}(\theta, \mu, \rho, q)\right]+R F(\theta, \mu, \rho, .)(q) \\
& +\widehat{\nu}\left(\alpha_{\theta}-c\right) \widehat{F}\left(\theta, \mu, \rho, \alpha_{\theta}-c\right)-R F(\theta, \mu, \rho, .)\left(\alpha_{\theta}-c\right) .
\end{aligned}
$$

Let us recall that $(H)$ implies the existence of $\gamma_{0}^{*}(\theta) \geq 0$ such that $\varphi\left(\gamma_{0}^{*}(\theta)\right)=\theta(c f$. (1.23) and (1.24)). Therefore taking $q=\gamma_{0}^{*}(\theta)$ in (3.52) brings to:

$$
\begin{aligned}
& \widehat{\nu}\left(\alpha_{\theta}-c\right) \widehat{F}\left(\theta, \mu, \rho, \alpha_{\theta}-c\right)-R F(\theta, \mu, \rho, .)\left(\alpha_{\theta}-c\right) \\
& =-\frac{1}{2} C_{\theta}\left(\gamma_{0}^{*}(\theta)\right)\left(\widehat{F}_{0}\left(\theta, \mu, \rho, \gamma_{0}^{*}(\theta)\right)+\widehat{F}_{1}\left(\theta, \mu, \rho, \gamma_{0}^{*}(\theta)\right)\right)-R F(\theta, \mu, \rho, .)\left(\gamma_{0}^{*}(\theta)\right) .
\end{aligned}
$$

First we determine $\widehat{F}_{0}\left(\theta, \mu, \rho, \gamma_{0}^{*}(\theta)\right)$ resp. $\widehat{F}_{1}\left(\theta, \mu, \rho, \gamma_{0}^{*}(\theta)\right)$ by using (3.34) resp. (3.43). Then relations (3.53) and (3.52) imply directly (2.17).

Step 2. Let $\nu_{n}$ be the finite measure on $\mathbb{R}$ :

$$
\nu_{n}(\mathrm{~d} y):=\nu_{\mid \mathbb{R} \backslash]-\frac{1}{n}, \frac{1}{n}[}(\mathrm{d} y) \quad \forall n \geq 1
$$

Let $F_{n}$ be the Laplace transform of $\left(T_{x}^{n}, K_{x}^{n}\right)$ :

$$
F_{n}(\theta, \mu, \rho, x)=\mathbb{E}\left(\mathrm{e}^{-\theta T_{x}^{n}-\mu K_{x}^{n}-\rho L_{x}^{n}} \mathbb{1}_{\left\{T_{x}^{n}<\infty\right\}}\right)
$$

where $X_{t}^{n}=B_{t}-c_{0}+J_{t}^{n},\left(J_{t}^{n}, \quad t \geq 0\right)$ a compound Poisson process with Lévy measure $\nu_{n}, T_{x}^{n}=\inf \left\{t \geq 0, X_{t}^{n}>x\right\}$ and $K_{x}^{n}=X_{T_{x}^{n}}^{n}-x$. Since $\lim _{n \rightarrow \infty} T_{x}^{n}=T_{x}$ and $\lim _{n \rightarrow \infty} K_{x}^{n}=K_{x}$, we can therefore infer that (2.17) will result from a limit procedure.

\subsection{Proof of Theorem 2.8}

Let us recall that $F(\theta, \mu, \rho,$.$) resp. \widehat{F}(\theta, \mu, \rho,$.$) have been defined by (2.9) resp. (0.6).$

In the sequel, $B \in] 0, B_{\nu}$ [ is supposed to be as close as possible to $B_{\nu}$. 
Step 1. We replace $F(\theta, \mu, \rho,$.$) by \widetilde{F}(\theta, \mu, \rho,$.$) ; an equation associated with \widetilde{F}(\theta, \mu, \rho,$.$) .$ We extend continuously $F(\theta, \mu, \rho,$.$) to the whole line as follows:$

$$
\widetilde{F}(\theta, \mu, \rho, x):=F(\theta, \mu, \rho, x) \mathbb{1}_{[0 ; \infty[}(x)+(1+x) \mathbb{1}_{[-1 ;+0]}(x), \quad \forall x \in \mathbb{R} .
$$

Let $\widehat{\widetilde{F}}(\theta, \mu, \rho,$.$) be the Laplace transform of \widetilde{F}(\theta, \mu, \rho,$.$) :$

$$
\widehat{\widetilde{F}}(\theta, \mu, \rho, q):=\int_{-\infty}^{\infty} \mathrm{e}^{-q x} \widetilde{F}(\theta, \mu, \rho, x) \mathrm{d} x .
$$

The advantage of using $\widetilde{F}(\theta, \mu, \rho,$.$) instead of F(\theta, \mu, \rho,$.$) lies in the fact that we shall prove below in Lemma 3.5,$ that $t \rightarrow \widehat{\widetilde{F}}\left(\theta, \mu, \rho, q_{1}+i t\right)$ is an integrable function on $\mathbb{R}$, for any $q_{1}$ in $] 0, \beta_{\theta}\left[\right.$ ( $\beta_{\theta}$ being defined in Prop. 2.7). Since $\int_{-1}^{0}(1+x) \mathrm{e}^{-q x} \mathrm{~d} x=\frac{\mathrm{e}^{q}-1-q}{q^{2}},(2.17)$ implies:

$$
\begin{aligned}
\widehat{\widetilde{F}}(\theta, \mu, \rho, q)= & \frac{\mathrm{e}^{q}-1-q}{q^{2}} \\
& +\frac{1}{\varphi(q)-\theta}\left(\frac{q-\gamma_{0}^{*}(\theta)}{2}+\int_{0}^{\infty}\left[\frac{\mathrm{e}^{-(q+\rho) y}-\mathrm{e}^{-\mu y}}{q+\rho-\mu}-\frac{\mathrm{e}^{-\left(\gamma_{0}^{*}(\theta)+\rho\right) y}-\mathrm{e}^{-\mu y}}{\gamma_{0}^{*}(\theta)+\rho-\mu}\right] \nu(\mathrm{d} y)\right. \\
& \left.+R F(\theta, \mu, \rho, .)(q)-R F(\theta, \mu, \rho, .)\left(\gamma_{0}^{*}(\theta)\right)\right) .
\end{aligned}
$$

We may observe that

$$
\begin{aligned}
& \frac{\varphi(q)-\theta}{q+\gamma_{0}^{*}(\theta)}=\frac{\varphi(q)-\varphi\left(\gamma_{0}^{*}(\theta)\right)}{q+\gamma_{0}^{*}(\theta)} \\
& =\frac{q-\gamma_{0}^{*}(\theta)}{2}+c \frac{q-\gamma_{0}^{*}(\theta)}{q+\gamma_{0}^{*}(\theta)}+\int_{-\infty}^{\infty} \frac{\mathrm{e}^{-q y}-\mathrm{e}^{-\gamma_{0}^{*}(\theta) y}+\left(q-\gamma_{0}^{*}(\theta)\right) y \mathbb{1}_{\{|y|<1\}}}{q+\gamma_{0}^{*}(\theta)} \nu(\mathrm{d} y),
\end{aligned}
$$

and

$$
\frac{\mathrm{e}^{q}-1-q}{q^{2}}+\frac{1}{q+\gamma_{0}^{*}(\theta)}=\frac{\mathrm{e}^{q}-1}{q^{2}}-\frac{\gamma_{0}^{*}(\theta)}{q\left(q+\gamma_{0}^{*}(\theta)\right)} .
$$

Consequently, for any $q$, so that $\operatorname{Re} q>0$ :

$$
\begin{aligned}
\widehat{\widetilde{F}}(\theta, \mu, \rho, q) & =\frac{\mathrm{e}^{q}-1}{q^{2}}-\frac{\gamma_{0}^{*}(\theta)}{q\left(q+\gamma_{0}^{*}(\theta)\right)} \\
& +\frac{1}{\varphi(q)-\theta}\left[-c \frac{q-\gamma_{0}^{*}(\theta)}{q+\gamma_{0}^{*}(\theta)}-\int_{-\infty}^{\infty} \frac{\mathrm{e}^{-q y}-\mathrm{e}^{-\gamma_{0}^{*}(\theta) y}+\left(q-\gamma_{0}^{*}(\theta)\right) y \mathbb{1}_{\{|y|<1\}}}{q(\mathrm{~d} y)}\right. \\
& +\int_{0}^{\infty}\left[\frac{\mathrm{e}^{-(q+\rho) y}-\mathrm{e}^{-\mu y}}{q+\rho-\mu} \nu(\mathrm{d} y)-\frac{\mathrm{e}^{-\left(\gamma_{0}^{*}(\theta)+\rho\right) y}-\mathrm{e}^{-\mu y}}{\gamma_{0}^{*}(\theta)+\rho-\mu}\right] \nu(\mathrm{d} y) \\
& \left.+R F(\theta, \mu, \rho, .)(q)-R F(\theta, \mu, \rho, .)\left(\gamma_{0}^{*}(\theta)\right)\right]
\end{aligned}
$$

It is clear that (1.11) implies that $R F(\theta, \mu, \rho,$.$) (defined by (2.16))$ is an entire function on $\mathbb{C}$ and all the integrals in (3.61) are holomorphic at least in $D_{-B_{\nu}}$. Therefore, the right hand-side of (3.61) is the meromorphic extension of $\widehat{\widetilde{F}}(\theta, \mu, \rho,$.$) to D_{-B_{\nu}}$. 
Before ending step 1 , we may remark that if $\theta>0$ or $\theta=0$ and $\mathbb{E}\left(X_{1}\right) \neq 0, \widehat{\widetilde{F}}(\theta, \mu, \rho,$.$) and \widehat{F}(\theta, \mu, \rho,$.$) are$ holomorphic in a neighborhood of $q=\gamma_{0}^{*}(\theta)$. Although $\gamma_{0}^{*}(\theta)$ is a zero of $\varphi_{\theta}$, this value is a false singularity for $\widehat{\widetilde{F}}(\theta, \mu, \rho,$.$) . But if \theta=0$ and $\mathbb{E}\left(X_{1}\right)=0$, then $\gamma_{0}^{*}(0)=0$ is a pole for $\widehat{\widetilde{F}}(0, \mu, \rho,$.$) .$

Step 2. $t \rightarrow \widehat{\widetilde{F}}\left(\theta, \mu, \rho, q_{1}+i t\right)$ belongs to $\mathbb{L}^{1}(\mathbb{R})$.

We may consider $\theta$ as a fixed element in $[0, \kappa]$ and $\beta_{\theta}>0$ as being given by Proposition 2.7. In addition, let $\left.q_{1} \in\right] 0, \beta_{\theta}[$.

Lemma 3.5. Under $(H)$ and (2.23), the function $t \rightarrow \widehat{\widetilde{F}}\left(\theta, \mu, \rho, q_{1}+i t\right)$ belongs to $\mathbb{L}^{1}(\mathbb{R})$.

So that to prove Lemma 3.5, we may begin with enumerating a few technical inequalities. These relations will be also used in the sequel.

Lemma 3.6. Let $\theta_{1}<\theta_{2}$. Let us suppose (2.23). Then:

(i) $\exists k>0$ so that for any $q$ which satisfies $\operatorname{Re} q \in\left[\theta_{1}, \theta_{2}\right]$, we have:

$$
\begin{aligned}
& \left|\int_{-1}^{1}\left(\mathrm{e}^{-q y}-1+q y \mathbb{1}_{\{|y|<1\}}\right) \nu(\mathrm{d} y)\right| \leq k|q|, \\
& \left|\int_{-1}^{0}\left(\mathrm{e}^{-q y}-1+q y \mathbb{1}_{\{|y|<1\}}\right) \nu(\mathrm{d} y)\right| \leq k|q|, \\
& \left|\int_{0}^{1}\left(\mathrm{e}^{-q y}-1+q y \mathbb{1}_{\{|y|<1\}}\right) \nu(\mathrm{d} y)\right| \leq k|q|,
\end{aligned}
$$

(ii) $\forall d>0, \quad \exists k_{0}>0 \quad$ such that $\sup _{\operatorname{Re} q \leq d}\left|\int_{-\infty}^{-1} \mathrm{e}^{-q y} \nu(\mathrm{d} y)\right| \leq k_{0}$,

(iii) $\forall A>0 \quad \exists k_{1}>0$ such that $\forall q$ which satisfies : $\left\{\begin{array}{l}-B \leq \operatorname{Re} q \leq A \\ |\operatorname{Im} q| \geq R_{0}\end{array}\right.$,

$$
\begin{aligned}
& \left|\int_{-\infty}^{\infty}\left(\mathrm{e}^{-q y}-1+q y \mathbb{1}_{\{|y|<1\}}\right) \nu(\mathrm{d} y)\right| \leq k_{1}(1+|q|), \\
& \frac{1}{k_{1}}|q|^{2} \leq|\varphi(q)| \leq k_{1}|q|^{2}
\end{aligned}
$$

(iv) $\forall h \in \mathbb{R}, \quad \sup _{\operatorname{Re} q \leq h}|R F(\theta, \mu, \rho, q)|<\infty$.

Proof of Lemma 3.6. The proof of Lemma 3.6 may be directly infered from (2.23).

Proof of Lemma 3.5. Proposition 2.7 tells us that $\varphi_{\theta}$ has no zero in the strip $\left\{q \in \mathbb{C} ; 0<\operatorname{Re} q<\beta_{\theta}\right\}$. So, if $\left.q_{1} \in\right] 0, \beta_{\theta}\left[, \varphi\left(q_{1}+i t\right)-\theta\right.$ never cancels and (3.61) implies that $t \rightarrow \widehat{\widetilde{F}}\left(\theta, \mu, \rho, q_{1}+i t\right)$ is a continuous function. Let us focus on (3.61). Lemma 3.6 implies that all the numerators in (3.61) are bounded on the line $\left\{q_{1}+i t ; t \in \mathbb{R}\right\}$, and the denominators are smaller than $C\left|q^{2}\right|$ for some $C>0$ and $|q|$ large. This proves $t \rightarrow \widehat{\widetilde{F}}\left(\theta, \mu, \rho, q_{1}+i t\right)$ belongs to $\mathbb{L}^{1}(\mathbb{R})$. 
Step 3. Proof of the asymptotic development (2.25), through the Mellin Fourier inverse transform.

Proposition 2.7 provides the existence of $\kappa>0$ and $B$ so that for any $\theta \in[0, \kappa], \varphi_{\theta}$ does not cancel on $\{-B+i t ; t \in \mathbb{R}\}$ and $B \neq \gamma_{0}^{*}(\theta)$.

Let $0<q_{1}<\beta_{\theta}$. Since $t \rightarrow \widehat{\widetilde{F}}\left(\theta, \mu, \rho, q_{1}+i t\right)$ belongs to $\mathbb{L}^{1}(\mathbb{R})$ (cf. Lem. 3.5), we may use the Mellin Fourier inverse transform (see for instance [19], p. 232-239). So, for any $x \geq 0$ :

$$
\mathrm{e}^{-q_{1} x} \widetilde{F}(\theta, \mu, \rho, x)=\mathrm{e}^{-q_{1} x} F(\theta, \mu, \rho, x)=\frac{1}{2 \pi} \int_{-\infty}^{\infty} \mathrm{e}^{i t x} \widehat{\widetilde{F}}\left(\theta, \mu, \rho, q_{1}+i t\right) \mathrm{d} t,
$$

hence

$$
F(\theta, \mu, \rho, x)=\frac{1}{2 \pi} \int_{-\infty}^{\infty} \mathrm{e}^{\left(q_{1}+i t\right) x} \widehat{\widetilde{F}}\left(\theta, \mu, \rho, q_{1}+i t\right) \mathrm{d} t=-\frac{i}{2 \pi} \int_{\Gamma_{q_{1}}} \mathrm{e}^{z x} \widehat{\widetilde{F}}(\theta, \mu, \rho, z) \mathrm{d} z,
$$

where $\Gamma_{q_{1}}$ is the path:

$$
\Gamma_{q_{1}}:=\left\{z=q_{1}+i t \quad \text { such that } t \in \mathbb{R}, \quad t \text { increasing }\right\} .
$$

In Proposition 2.7, it has been proved that there is $R_{1}>R_{0}$, so that $\varphi_{\theta}$ has no zero in the two half-strips $\left\{q \in \mathbb{C} ; \quad-B \leq \operatorname{Re} q<\beta_{\theta}\right.$ and $\left.|\operatorname{Im} q|>R_{1}\right\}$. In particular $\widehat{\widetilde{F}}(\theta, \mu, \rho,$.$) is holomorphic in this domain.$

Let $\Gamma_{-B, q_{1}, R}$ be the rectangular path (see Fig. 3):

$$
\Gamma_{-B, q_{1}, R}:=\Gamma_{q_{1}, R} \cup \Gamma_{R} \cup \Gamma_{-B, R} \cup \Gamma_{-R}
$$

where:

$$
\begin{aligned}
& \Gamma_{q_{1}, R}:=\left\{q_{1}+i t ; \quad|t| \leq R, \quad t \text { growing }\right\} \\
& \Gamma_{R}:=\left\{t+i R ; \quad-B \leq t \leq q_{1}, \quad t \text { decreasing }\right\}, \\
& \Gamma_{-B, R}:=\{-B+i t ; \quad|t| \leq R, \quad t \text { decreasing }\} \\
& \Gamma_{-R}:=\left\{t-i R ; \quad-B \leq t \leq q_{1}, \quad t \text { growing }\right\} .
\end{aligned}
$$

If the residual theorem is applied to the meromorphic extension of $z \rightarrow \mathrm{e}^{z x} \widehat{\widetilde{F}}(\theta, \mu, \rho, z)$ to $D_{-} B_{\nu}$, the result is for any $R>R_{1}$ :

$$
\int_{\Gamma_{-B, q_{1}, R}} \mathrm{e}^{z x} \widehat{\widetilde{F}}(\theta, \mu, \rho, z) \mathrm{d} z=2 i \pi\left[C_{0}(\theta, \mu, \rho,) \mathrm{e}^{-\gamma_{0}(\theta) x}+\sum_{i=1}^{p} a_{i}\left(C_{i}(\theta, \mu, \rho, x) \mathrm{e}^{-\gamma_{i}(\theta) x}+\overline{C_{i}}(\theta, \mu, \rho, x) \mathrm{e}^{-\overline{\gamma_{i}}(\theta) x}\right)\right]
$$

where $a_{i}=\frac{1}{2}$ if $-\gamma_{i}(\theta)$ is a real number and $a_{i}=1$ otherwise, and:

$$
\begin{aligned}
& C_{0}(\theta, \mu, \rho):=\operatorname{Res}\left(\widehat{\widetilde{F}}(\theta, \mu, \rho, z) ;-\gamma_{0}(\theta)\right), \\
& C_{i}(\theta, \mu, \rho, x):=\mathrm{e}^{\gamma_{i}(\theta) x} \operatorname{Res}\left(\mathrm{e}^{z x} \widehat{\widetilde{F}}(\theta, \mu, \rho, z) ;-\gamma_{i}(\theta)\right),
\end{aligned}
$$

where $\operatorname{Res}(f(z) ; \gamma)$ denotes the residual of $f$ at point $\gamma$. Let us notice that $(3.78)$ is valid since $-\gamma_{0}(\theta)$ is a simple pole of $\widehat{\widetilde{F}}(\theta, \mu, \rho,$.$) .$ 


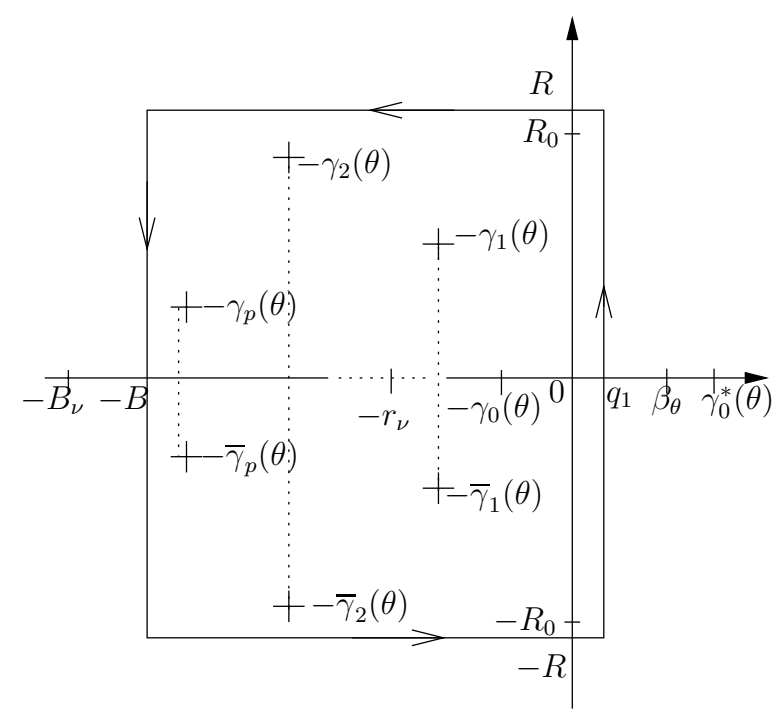

Figure 3 . The path $\Gamma_{-B, q_{1}, R}$.

Since $z \rightarrow \frac{\mathrm{e}^{z}-1-z}{z^{2}}$ has an holomorphic extension to the whole plan $\mathbb{C}$, the identity (3.56) implies that:

$$
\begin{aligned}
& C_{0}(\theta, \mu, \rho)=\operatorname{Res}\left(\widehat{F}(\theta, \mu, \rho, z) ;-\gamma_{0}(\theta)\right), \\
& C_{i}(\theta, \mu, \rho, x)=\mathrm{e}^{\gamma_{i}(\theta)} \operatorname{Res}\left(\mathrm{e}^{z x} \widehat{F}(\theta, \mu, \rho, z) ;-\gamma_{i}(\theta)\right) \quad \forall i \in\{1, \cdots, p\} .
\end{aligned}
$$

We observe that $\overline{C_{i}}(\theta, \mu, \rho, x)=\mathrm{e}^{\overline{\gamma_{i}}(\theta) x} \operatorname{Res}\left(\mathrm{e}^{z x} \widehat{F}(\theta, \mu, \rho, z) ;-\overline{\gamma_{i}}(\theta)\right)$. We will prove below in Section 4.5, that $x \rightarrow C_{i}(\theta, \mu, \rho, x)$ is a polynomial function (see the formula (4.30)). Since $z \rightarrow \mathrm{e}^{z x} \widehat{\widetilde{F}}(\theta, \mu, \rho, z)$ belongs to $\mathbb{L}^{1}(\mathbb{R})$, we come to $(c f .(3.69))$ :

$$
\begin{aligned}
& F(\theta, \mu, \rho, x)=-\frac{i}{2 \pi} \lim _{R \rightarrow \infty} \int_{\Gamma_{q_{1}, R}} \mathrm{e}^{z x} \widehat{\widetilde{F}}(\theta, \mu, \rho, z) \mathrm{d} z \\
&=-\frac{i}{2 \pi} \lim _{R \rightarrow \infty}\left[\int_{\Gamma_{-B, q_{1}, R}} \mathrm{e}^{z x} \widehat{\widetilde{F}}(\theta, \mu, \rho, z) \mathrm{d} z-\int_{\Gamma_{R}} \mathrm{e}^{z x} \widehat{\widetilde{F}}(\theta, \mu, \rho, z) \mathrm{d} z\right. \\
&\left.\quad \int_{\Gamma_{-B, R}} \mathrm{e}^{z x} \widehat{\widetilde{F}}(\theta, \mu, \rho, z) \mathrm{d} z-\int_{\Gamma_{-R}} \mathrm{e}^{z x} \widehat{\widetilde{F}}(\theta, \mu, \rho, z) \mathrm{d} z\right] .
\end{aligned}
$$

We claim that in the right hand-side of (3.82), the limits of the second term and the fourth one are null. As for the third limit, we have:

$$
\lim _{R \rightarrow \infty} \int_{\Gamma_{-B, R}} \mathrm{e}^{z x} \widehat{\widetilde{F}}(\theta, \mu, \rho, z) \mathrm{d} z=\mathrm{O}\left(\mathrm{e}^{-B x}\right)
$$

where $\mathrm{O}$ is uniform with respect to $\theta \in[0, \kappa], \mu \in \mathbb{R}_{+}$and $\rho \in \mathbb{R}_{+}$.

Hence, as $x \rightarrow \infty$ :

$$
F(\theta, \mu, \rho, x)=C_{0}(\theta, \mu, \rho) \mathrm{e}^{-\gamma_{0}(\theta)}+\sum_{i=1}^{p} a_{i}\left[C_{i}(\theta, \mu, \rho, x) \mathrm{e}^{-\gamma_{i}(\theta) x}+\overline{C_{i}}(\theta, \mu, \rho, x) \mathrm{e}^{-\overline{\gamma_{i}}(\theta) x}\right]+\mathrm{O}\left(\mathrm{e}^{-B x}\right) .
$$


Remark 3.7. Let us determine the sign of $C_{0}(\theta, \mu, \rho)$. The asymptotic expansion (2.25) implies that:

$$
\lim _{x \rightarrow \infty} \mathrm{e}^{\gamma_{0}(\theta) x} F(\theta, \mu, \rho, x)=C_{0}(\theta, \mu, \rho) \geq 0 .
$$

The residual $C_{0}(\theta, \mu, \rho)$ at $-\gamma_{0}(\theta)$ of $F(\theta, \mu, \rho,$.$) cannot cancel because -\gamma_{0}(\theta)$ is a single pole of $\widehat{F}(\theta, \mu, \rho,$. when $\theta>0$ or $\theta=0$ and $\mathbb{E}\left(X_{1}\right) \neq 0$.

If $\theta=0$ and $c=\mathbb{E}\left(J_{1}\right)$, it is easy to see that $C_{0}(0, \mu, \rho) \neq 0$ ( $c f$. item c) iii) in Section 4.5 below).

We will give in Section 4.5 some complements related to the calculation of the coefficients $C_{i}(\theta, \mu, \rho, x)$.

\subsection{Proof of Theorem 2.10}

Let us recall that the ruin probability is the function:

$$
F(x):=F(0,0,0, x)=\mathbb{P}\left(T_{x}<\infty\right)
$$

Let us denote $\widehat{F}$ the Laplace transform of $F$ :

$$
\widehat{F}(q):=\int_{0}^{\infty} \mathrm{e}^{-q x} F(x) \mathrm{d} x \quad \forall q \in \mathbb{C}, \operatorname{Re}(q)>0 .
$$

In this section, it is only assumed that the Lévy measure $\nu$ satisfies (2.30) and (2.31).

The proof will be divided into five parts.

Step 1. We will prove that we only need to consider a Lévy measure $\nu$ whose support is included in $[-k, \infty[$, for some (finite) $k \geq 0$.

The assumption (2.30) implies that there is $k>0$ such that

$$
\int_{-k}^{\infty} \mathbb{1}_{\{|y| \geq 1\}} y \nu(\mathrm{d} y)<c .
$$

Let $\left(X_{t}^{k}, t \geq 0\right)$ be a Lévy process with decomposition:

$$
X_{t}^{k}:=B_{t}-c_{0} t+J_{t}^{k} \quad \forall t \geq 0,
$$

where $\left(J_{t}^{k}, t \geq 0\right)$ is a pure jump process independent from $\left(B_{t}, t \geq 0\right)$ and with Lévy measure: $\nu_{k}:=\nu_{\left.\right|_{[-k, \infty}}$. In addition, it can be supposed that the processes $\left(J_{t}^{k}, t \geq 0\right)$ and $\left(J_{t}, t \geq 0\right)$ are defined on the same probability space. Since $\nu-\nu_{k}$ is a non-negative measure whose support is included in $\left.]-\infty,-k\right]$, then $J_{t} \leq J_{t}^{k}, \forall t \geq 0$. Consequently $X_{t} \leq X_{t}^{k}, \forall t \geq 0$ a.s., and $T_{x}^{k} \leq T_{x}, \forall x \geq 0$ where

$$
T_{x}^{k}:=\inf \left\{t \geq 0 / X_{t}^{k}>x\right\}
$$

As a result,

$$
\forall x \geq 0 \quad F(x) \leq F^{k}(x):=\mathbb{P}\left(T_{x}^{k}<\infty\right) .
$$

Note that the relation (3.88) implies that $\nu_{k}$ satisfies (2.30) and (2.31). This proves the claim. 
Step 2. The aim is to prove that we can suppose that the support of $\nu$ is included in $[-k,-1] \cup[0, \infty[$. It is clear that the relation (2.30) implies that there is $\varepsilon>0$ so that

$$
-c+\varepsilon+\int_{[-k, \infty[} y \mathbb{1}_{\{|y| \geq 1\}} \nu(\mathrm{d} y)<0 .
$$

Let $\nu^{(1)}$ be the Lévy measure: $\nu^{(1)}(\mathrm{d} y):=\mathbb{1}_{]-1,0}\left[(y) \nu(\mathrm{d} y)\right.$. Consider $X^{(1)}$ the Lévy process whose function $\varphi^{(1)}$ is defined as follows:

$$
\varphi^{(1)}(q):=\varepsilon q+\int_{\mathbb{R}}\left(\mathrm{e}^{-q y}-1+q y \mathbb{1}_{\{|y|<1\}}\right) \nu^{(1)}(\mathrm{d} y)=\varepsilon q+\int_{]-1,0[}\left(\mathrm{e}^{-q y}-1+q y\right) \nu(\mathrm{d} y) .
$$

Consequently $\mathbb{E}\left(X_{1}^{(1)}\right)=-\varepsilon<0$.

Since $\varphi^{(1)}(q) \geq-q \varepsilon$ for any $\left.\left.q \in\right]-\infty, 0\right]$, then $\lim _{q \rightarrow-\infty} \varphi^{(1)}(q)=\infty$. Note that the graph of $\varphi^{(1)}$ corresponds to Figure 1, case a). As a result, there exist $\gamma^{(1)}>0$ such that $\varphi^{(1)}\left(-\gamma^{(1)}\right)=0$. According to [2] there exists $C^{(1)}>0$ such that

where $T_{x}^{(1)}:=\inf \left\{t \geq 0, X_{t}^{(1)}>x\right\}$.

$$
\mathbb{P}\left(T_{x}^{(1)}<\infty\right) \leq C^{(1)} \mathrm{e}^{-\gamma^{(1)} x}, \quad x \geq 0
$$

Next let $\nu^{(2)}(\mathrm{d} y):=\mathbb{1}_{[-k,-1] \cup] 0, \infty[}(y) \nu(\mathrm{d} y)$, and $X^{(2)}$ the Lévy process associated with

$$
\varphi^{(2)}(q):=\frac{q^{2}}{2}+(c-\varepsilon) q+\int_{\mathbb{R}}\left(\mathrm{e}^{-q y}-1+q y \mathbb{1}_{\{|y| \geq 1\}}\right) \nu^{(2)}(\mathrm{d} y)
$$

Consequently:

$$
\mathbb{E}\left(X_{1}^{(2)}\right)=-(c-\varepsilon)+\int_{\mathbb{R}} y \mathbb{1}_{\{|y| \geq 1\}} \nu^{(2)}(\mathrm{d} y)=-c+\varepsilon+\int_{\mathbb{R}} y \mathbb{1}_{\{|y| \geq 1\}} \nu(\mathrm{d} y)<0 .
$$

In addition suppose that $X^{(1)}$ and $X^{(2)}$ are independent. Since $\varphi=\varphi^{(1)}+\varphi^{(2)}$, therefore $X$ is distributed as $X^{(1)}+X^{(2)}$. Let us note that:

$$
\left\{T_{x / 3}^{(1)}=\infty\right\} \cap\left\{T_{x / 3}^{(2)}=\infty\right\} \subset\left\{X_{t}^{(1)}+X_{t}^{(2)}<x\right\}, \quad x>0 .
$$

Applying moreover (3.93) we get:

$$
\mathbb{P}\left(T_{x}<\infty\right) \leq \mathbb{P}\left(T_{x / 3}^{(1)}<\infty\right)+\mathbb{P}\left(T_{x / 3}^{(2)}<\infty\right) \leq C^{(1)} \mathrm{e}^{-\gamma^{(1)} x / 3}+\mathbb{P}\left(T_{x / 3}^{(2)}<\infty\right)
$$

This inequality implies that the rate of decay of $x \mapsto \mathbb{P}\left(T_{x}<\infty\right)$ is polynomial as soon as $x \mapsto \mathbb{P}\left(T_{x}^{(2)}<\infty\right)$ enjoys the same asymptotic behavior.

In the sequel we can suppose that (2.30) and (2.31) hold and the support of $\nu$ is included in $[-k,-1] \cup[0, \infty[$, for some $k>0$.

Step 3. $F$ belongs to $\mathbb{L}^{1}\left(\mathbb{R}_{+}\right)$.

We will first prove that:

$$
\sup _{0<q \leq q_{0}}|\widehat{F}(q)|<\infty, \quad \text { for some } q_{0}>0 .
$$

By taking the limit $\theta, \mu, \rho \rightarrow 0$ in (2.17), we may easily obtain:

$$
\widehat{F}(q)=\frac{1}{\varphi(q)}\left(\frac{q}{2}+\frac{1}{q} \int_{0}^{\infty}\left(\mathrm{e}^{-q y}-1+q y\right) \nu(\mathrm{d} y)+R F(q)\right) .
$$


Let us determine the asymptotic behavior of the numerator and the denominator as $q \rightarrow 0$. Let us begin with the denominator:

$$
\varphi(q) \sim q \varphi^{\prime}(0)=q\left(c-\int_{\mathbb{R}} \mathbb{1}_{\{|y| \geq 1\}} y \nu(\mathrm{d} y)\right) \quad \text { as } q \rightarrow 0 .
$$

Next, we consider the numerator. We have:

$$
\frac{q}{2}+\frac{1}{q} \int_{0}^{\infty}\left(\mathrm{e}^{-q y}-1+q y\right) \nu(\mathrm{d} y) \sim \frac{q}{2}\left[1+\int_{0}^{\infty} y^{2} \nu(\mathrm{d} y)\right] \quad \text { as } q \rightarrow 0 .
$$

Since the support of $\nu$ is included in $[-k,-1] \cup[0, \infty[$, then $R F(q)$ can be simplified:

$$
R F(q)=\int_{-k}^{-1} \nu(\mathrm{d} y) \int_{0}^{-y}\left(\mathrm{e}^{-q(b+y)}-1\right) F(b) \mathrm{d} b .
$$

But $R F(0)=0$ and the derivative of $R F(q)$ is bounded, then $|R F(q)| \leq C q$, for any $0 \leq q \leq q_{0}$. (3.94) follows immediately.

It is now easy to check that $F$ is in $\mathbb{L}^{1}\left(\mathbb{R}_{+}\right)$. The function $F$ is non-negative, then the monotone convergence theorem implies:

$$
\int_{0}^{\infty} F(x) \mathrm{d} x=\lim _{q \rightarrow 0} \int_{0}^{\infty} \mathrm{e}^{-q x} F(x) \mathrm{d} x \leq \sup _{0<q \leq q_{0}} \widehat{F}(q)<\infty .
$$

As a result $F$ is integrable.

\section{Step 4. Definition of $\widetilde{F}$.}

The function $F$ can be extended to the whole line, setting $F(x)=0$, for any $x \leq 0$. However $F$ may have a jump at 0 . Let $\widetilde{F}$ be the following continuous extension of $F$ :

$$
\widetilde{F}(x):=F(x) \mathbb{1}_{[0 ; \infty[}(x)+(1+x) \mathbb{1}_{[-1 ; 0]}(x), \quad \forall x \in \mathbb{R} .
$$

Let $q \rightarrow \widehat{\widetilde{F}}(i q)$ be the Fourier transform of $\widetilde{F}$ :

$$
\widehat{\widetilde{F}}(i q)=\int_{-\infty}^{\infty} \mathrm{e}^{-i q x} \widetilde{F}(x) \mathrm{d} x=\int_{-1}^{\infty} \mathrm{e}^{-i q x} \widetilde{F}(x) \mathrm{d} x \quad \forall q \in \mathbb{R} .
$$

Step 5. $q \rightarrow \widehat{\widetilde{F}}(i q)$ is in $\mathbb{L}^{1}(\mathbb{R})$.

Since $F \in \mathbb{L}^{1}\left(\mathbb{R}_{+}\right)$, then $\widetilde{F} \in \mathbb{L}^{1}(\mathbb{R})$ and $q \mapsto \widehat{\widetilde{F}}(i q)$ is continuous.

So, if we establish:

$$
|\widehat{\widetilde{F}}(i q)| \leq \frac{C}{1+q^{2}}
$$

then $\widehat{\widetilde{F}}$ will be an element of $\mathbb{L}^{1}(\mathbb{R})$. It is proved in Proposition 2.7, that $\varphi(i q)=0, q \in \mathbb{R}$ iff $q=0$. Therefore we are allowed to replace $q$ by $i q$ in (3.95). By using the identity:

$$
\int_{-1}^{0}(1+x) \mathrm{e}^{-i q x} \mathrm{~d} x=-\frac{\mathrm{e}^{i q}-1-i q}{q^{2}},
$$

and (3.94) we may deduce: 
(1) (3.102) holds for any $|q| \leq q_{0}$ (for some $q_{0}>0$ );

(2) $\widehat{\widetilde{F}}(i q)$ may be written as follows:

$$
\widehat{\widetilde{F}}(i q)=\frac{1-\mathrm{e}^{i q}}{q^{2}}+\frac{1}{\varphi(i q)}\left[-c-\int_{1}^{\infty} y \nu(\mathrm{d} y)+\frac{i}{q} \int_{-k}^{-1}\left(\mathrm{e}^{-i q y}-1\right) \nu(\mathrm{d} y)+R F(i q)\right] .
$$

The inequality (3.102) will be a direct consequence of following estimates:

$$
\begin{aligned}
& \varphi(i q) \sim-\frac{q^{2}}{2}\left(\int_{\mathbb{R}} y^{2} \mathbb{1}_{\{|y|<1\}} \nu(\mathrm{d} y)\right) \quad(|q| \rightarrow \infty) \\
& \left|\int_{-k}^{0}\left(\mathrm{e}^{-i q y}-1\right) \nu(\mathrm{d} y)\right| \leq|q| k \nu([-k,-1]) \\
& |R F(i q)| \leq 2 k \nu([-k,-1]) .
\end{aligned}
$$

Step 6. The $n$ first derivatives of $\widehat{\widetilde{F}}$ belong to $\mathbb{L}^{1}(\mathbb{R})$.

Obviously any $k$ derivative of $q \rightarrow \frac{1-\mathrm{e}^{i q}}{q^{2}}$ is continuous, bounded by $\frac{C}{q^{2}},|q| \geq 1$, and therefore belongs to $\mathbb{L}^{1}(\mathbb{R})$. The second term in the right hand-side of (3.104) may be written this way: $\frac{N(q)}{\varphi(i q)}$.

By proceeding likewise step 5 and using (2.31) it can be proved that the $n$ first derivatives of $N$ are bounded. As a result $\left|\frac{N(q)}{\varphi(i q)}\right| \leq \frac{C}{q^{2}},|q| \geq 1$.

As for the asymptotic behavior of $\frac{N(q)}{\varphi(i q)}$ in a neighborhood of 0 , it can be proved through a similar reasoning that this ratio is bounded, for any $|q| \leq 1$.

Step 7. Proof of (2.32).

Since the $n$ derivatives of $q \rightarrow \widehat{\widetilde{F}}(i q)$ belong to $\mathbb{L}^{1}(\mathbb{R})$, then

$$
x^{n} \widetilde{F}(x)=\frac{i^{n}}{2 \pi} \int_{\mathbb{R}} \mathrm{e}^{i q x} \frac{d^{n}}{d q^{n}}(\widehat{\widetilde{F}}(i q)) \mathrm{d} q .
$$

This identity directly implies (2.32).

\section{Appendix}

\subsection{Rate of convergence in Theorem 2.1}

We would like to point out that the asymptotic development of $F(\theta, \mu, \rho, x)$ which is given by $(2.25)$ provides the rate of convergence of $\left(\frac{1}{\sqrt{x}}\left(T_{x}+\frac{x}{\varphi^{\prime}\left(-\gamma_{0}(0)\right)}\right)\right)$ to the Gaussian distribution. Let us suppose that $\mathbb{E}\left[X_{1}\right]>0$. Let $A_{x}$ be the distribution function of $\left(\frac{1}{\sqrt{x}}\left(T_{x}+\frac{x}{\varphi^{\prime}\left(-\gamma_{0}(0)\right)}\right)\right)$ and let $\widehat{A}_{x}$ be its characteristic function:

$$
\begin{array}{ll}
A_{x}(t)=\mathbb{P}\left(\frac{1}{\sqrt{x}}\left(T_{x}+\frac{x}{\varphi^{\prime}\left(-\gamma_{0}(0)\right)}\right) \leq t\right), \quad t \in \mathbb{R} \\
\widehat{A}_{x}(\theta)=\mathbb{E}\left[\mathrm{e}^{\frac{i \theta}{\sqrt{x}}\left(T_{x}+\frac{x}{\varphi^{\prime}\left(-\gamma_{0}(0)\right)}\right)}\right], & \theta \in \mathbb{R} .
\end{array}
$$

Thanks to $(2.25)$, it is not difficult to check that, if $x$ is large enough we obtain:

$$
\sup _{\theta>0}\left|\widehat{A}_{x}(\theta)-\widehat{A}(\theta)\right| \leq \frac{k \theta}{\sqrt{x}}
$$


Here $k$ is a positive constant and $\widehat{A}$ is the characteristic function of $\mathcal{N}\left(0 ;-\frac{\varphi^{\prime \prime}\left(-\gamma_{0}(0)\right)}{\varphi^{\prime 3}\left(-\gamma_{0}(0)\right)}\right)$.

Berry-Essen's inequalities (see for instance [15], p. 285) imply the existence of two positives constants $k_{1}$ and $k_{2}$ such that:

$$
\sup _{t \in \mathbb{R}}\left|A_{x}(t)-A(t)\right| \leq k_{1} \int_{0}^{a} \frac{k \theta}{\sqrt{x}} \frac{\mathrm{d} \theta}{\theta}+\frac{k_{2}}{a}, \quad \text { for any } a>0,
$$

where $A$ is the distribution function of the previous Gaussian distribution.

Choosing $a=x^{1 / 4}$, we get:

$$
\sup _{t \in \mathbb{R}}\left|A_{x}(t)-A(t)\right| \leq \frac{k_{3}}{x^{1 / 4}}, x \geq 1,
$$

for some $k_{3}>0$

\subsection{Stochastic interpretation of $w^{-}$and $w^{+}$}

We would like to give a stochastic interpretation of the probability measure $w^{-}$defined by $(2.2)$. Let $(K, L)$ be a two-dimensional r.v. with probability distribution $w^{-}$. Obviously the event $\{K=0\}(=\{L=0\}$ a.s.) occurs with probability $\frac{-\gamma_{0}(0)}{2 \mathbb{E}\left(X_{1}\right)}$. Conditionally on $\{L>0\}$, the distribution of $(K, L)$ is of type $\alpha\left(\mathrm{e}^{\gamma_{0}(0) l}-1\right) \mathbb{1}_{\{k>0 ; l>0\}} \nu_{l}(\mathrm{~d} k) \mathrm{d} l$ where $\nu_{l}$ is the positive measure defined in Theorem 2.1.

This leads us to consider the positive measure:

$$
w^{(\gamma)}(\mathrm{d} k, \mathrm{~d} l)=\alpha\left(\mathrm{e}^{\gamma l}-1\right) \mathbb{1}_{\{k>0 ; l>0\}} \nu_{l}(\mathrm{~d} k) \mathrm{d} l,
$$

in which $\gamma>0, \nu_{l}$ is the image of $\nu$ by $y \rightarrow y-l$. In addition, $\nu$ is a positive measure on $] 0 ; \infty[$ which satisfies:

$$
\int_{0}^{\infty}\left(\mathrm{e}^{\gamma k}-1-\gamma k\right) \nu(\mathrm{d} k)<\infty,
$$

here $\alpha$ is the normalization factor: $\alpha=\frac{\gamma}{\int_{0}^{\infty}\left(\mathrm{e}^{\gamma k}-1-\gamma k\right) \nu(\mathrm{d} k)}$.

Proposition 4.1. Let $\left(K^{*}, L^{*}\right)$ be a two dimensional r.v. with distribution $w^{(\gamma)}$ defined by (4.4). Then $L^{*}$ has a density function given by $\alpha\left(\mathrm{e}^{\gamma l}-1\right) \nu\left(\left[l, \infty[) \mathbb{1}_{\{l>0\}}\right.\right.$. Conditionally on $L^{*}=l$, the distribution of $S^{*}=L^{*}+K^{*}$ is $\frac{1}{\nu(l, \infty \infty)} \mathbb{1}_{\{s>l\}} \nu(\mathrm{d} s)$.

It is obvious that choosing $\gamma=\gamma_{0}(0)$ (resp. $\gamma=\gamma_{0}^{*}(0)$ ) allows to recover the probability measure $w^{-}$ (resp. $w^{+}$) introduced in (2.2) (resp. (2.5)). Consequently, Proposition 4.1 gives a stochastic interpretation of the limit law of $\left(K_{x}, L_{x}\right)$ as $x \rightarrow \infty$, in the case where $\nu(]-\infty ; 0[)=0$.

\subsection{Study of $\Lambda_{\theta}$}

To investigate uniqueness in (2.10), we will prove that $\Lambda_{\theta}$ is a contraction on the Banach space:

$$
\mathcal{B}_{\gamma}:=\left\{f: \mathbb{R}_{+} \rightarrow \mathbb{R} ; \sup _{x \in \mathbb{R}_{+}} \mathrm{e}^{\gamma x}|f(x)|<\infty\right\} \quad \gamma \geq 0 .
$$

$\mathcal{B}_{\gamma}$ is equipped with the norm:

$$
\|f\|_{\gamma}:=\sup _{x \in \mathbb{R}_{+}} \mathrm{e}^{\gamma x}|f(x)| .
$$

Theorem 4.2. Let us suppose that $\nu(\mathbb{R})<\infty$.

(i) For any $\theta \geq 0$, the operator $\Lambda_{\theta}$ defined by (2.14) is a linear and non-negative operator, whose norm is equal to $\frac{\lambda}{\lambda+\theta}$ in $L^{\infty}\left(\mathbb{R}_{+}\right)$. 
(ii) Let $\gamma \in\left[0, r_{\nu}\left[\right.\right.$ and $\theta>0$ or $\theta=0$ and $\mathbb{E}\left(X_{1}\right)<0$ (recall that $r_{\nu}$ has been defined by (1.13)). Then:

a) $\Lambda_{\theta}$ is a bounded operator from $\mathcal{B}_{\gamma}$ to $\mathcal{B}_{\gamma}$. More precisely:

$$
\left\|\Lambda_{\theta} f\right\|_{\gamma} \leq c_{\theta, \gamma}\|f\|_{\gamma} \quad \forall f \in \mathcal{B}_{\gamma}
$$

with

$$
c_{\theta, \gamma}=\frac{\widehat{\nu}(-\gamma)}{\widehat{\nu}(-\gamma)-\varphi(-\gamma)+\theta}
$$

and $\widehat{\nu}$ the Laplace transform of $\nu(c f(3.47))$.

b) There exists $\gamma \in] 0, r_{\nu}\left[\right.$ such that $\varphi(-\gamma)<\theta$. Therefore $\Lambda_{\theta}$ is a $\mathcal{B}_{\gamma}$-contraction since:

$$
0<c_{\theta, \gamma}<1
$$

Proof of Theorem 4.2.

(i) Relation (3.31) implies that $\Lambda_{\theta}$ is a non-negative operator.

It is easy to check that the function $\ell$ :

$$
\ell(x):=\mathbb{1}_{\{a+y \leq x ; a \leq x\}} \mathrm{e}^{-c_{0} a}\left(\mathrm{e}^{-\alpha_{\theta}|a|}-\mathrm{e}^{-(2 x-a) \alpha_{\theta}}\right)
$$

is increasing, then:

$$
\ell(x)<\ell(\infty)=\mathrm{e}^{-c_{0} a} \mathrm{e}^{-\alpha_{\theta}|a|} \quad \forall x \in \mathbb{R} .
$$

A straightforward calculation shows that $\Lambda_{\theta} h(x) \mid \leq \frac{\lambda}{\lambda+\theta}\|h\|_{\infty}$, for any $x \geq 0$.

If we take $h: x \rightarrow 1$, we have $\left\|\Lambda_{\theta} h\right\|_{\infty}=\frac{\lambda}{\lambda+\theta}$. Therefore, $\left\|\left|\Lambda_{\theta}\right|\right\|_{L^{\infty}\left(\mathbb{R}_{+}\right)}=\frac{\lambda}{\lambda+\theta}$.

(ii) Let $f$ be an element of $\mathcal{B}_{\gamma}$, then $|f(x)| \leq\|f\|_{\gamma} \mathrm{e}^{-\gamma x}, \forall x \geq 0$. It follows that:

$$
\left|\Lambda_{\theta} f(x)\right| \leq \frac{1}{\alpha_{\theta}}\|f\|_{\gamma} \mathrm{e}^{-\gamma x} \int_{-\infty}^{\infty} \nu(\mathrm{d} y) \int_{-\infty}^{(x-y) \wedge x} \mathrm{e}^{-c_{0} a}\left(\mathrm{e}^{-\alpha_{\theta}|a|}-\mathrm{e}^{-(2 x-a) \alpha_{\theta}}\right) \mathrm{e}^{\gamma(a+y)} \mathrm{d} a
$$

for any $\gamma \in\left[0, r_{\nu}[\right.$.

By using (4.12), we get:

$$
\left|\Lambda_{\theta} f(x)\right| \leq \frac{1}{\alpha_{\theta}}\|f\|_{\gamma} \mathrm{e}^{-\gamma x} \int_{-\infty}^{\infty} \nu(\mathrm{d} y) \mathrm{e}^{\gamma y}\left[\int_{-\infty}^{0} \mathrm{e}^{-\left(c_{0}-\alpha_{\theta}-\gamma\right) a} \mathrm{~d} a+\int_{0}^{\infty} \mathrm{e}^{-\left(c_{0}+\alpha_{\theta}-\gamma\right) a} \mathrm{~d} a\right]
$$

Computing the integral with respect to da, leads directly to (4.8).

Proposition 4.3. Let us assume $\nu(\mathbb{R})<\infty, r_{\nu}>0, \mu \geq 0, \theta>0$, or $\theta=0$ if $\mathbb{E}\left(X_{1}\right)<0$. Let $\gamma$ be in $\left[0, r_{\nu}[\right.$, such that $\varphi(-\gamma)<\theta$. Then the function $F(\theta, \mu, \rho,$.$) belongs to \mathcal{B}_{\gamma}$ and the equation (2.10) has a unique solution in $\mathcal{B}_{\gamma}$.

To prove Proposition 4.3, we need the following preliminary.

Lemma 4.4. Suppose either $\theta>0$, or $\theta=0$ if $\mathbb{E}\left(X_{1}\right)<0$, then for any $x>0$,

$$
\lim _{n \rightarrow \infty} \Lambda_{\theta}^{n} F(\theta, \mu, \rho, .)(x)=0 .
$$


Proof of Lemma 4.4 .

1) Suppose $\theta>0$. Since $F$ is bounded by 1 , and the norm of $\Lambda_{\theta}$ is $\frac{\lambda}{\lambda+\theta}$ (cf. Th. 4.2): $\left\|\Lambda_{\theta}^{n} F(\theta, \mu, \rho, .)\right\|_{\infty} \leq$ $\left(\frac{\lambda}{\lambda+\theta}\right)^{n}$. This proves (4.14).

2) Let us now turn to the case $\theta=0$ and $\mathbb{E}\left(X_{1}\right)<0$. By iterating the functional equation (2.10), we come to:

$$
F(\theta, \mu, \rho, x)=\sum_{p=0}^{n-1} \Lambda_{\theta}^{p}\left[\left(F_{0}+F_{1}\right)(\theta, \mu, \rho, .)\right](x)+\Lambda_{\theta}^{n} F(\theta, \mu, \rho, .)(x) .
$$

The $\mathcal{B}_{\gamma}$-norm of $\Lambda_{\theta}$ is strictly less than 1 , then the series in (4.15) converges. The remaining term $\Lambda_{\theta}^{n} F(\theta, \mu, \rho,).(x)$ converges in $\mathcal{B}_{\gamma}$ to some function $G(\theta, \mu, \rho, x)$. It is easy to check what follows:

a) $G(0, \mu, \rho,$.$) is a bounded and non negative function;$

b) $G(0, \mu, \rho,$.$) is a continuous function on [0, \infty[$;

c) $\lim _{x \rightarrow \infty} G(0, \mu, \rho, x)=0$;

d) $\Lambda_{0} G(0, \mu, \rho,)=.G(0, \mu, \rho,$.$) .$

Using $(i)$ in Theorem 4.2 leads to:

$$
G(0, \mu, \rho, x)=\Lambda_{0} G(0, \mu, \rho, .)(x) \leq\|G(0, \mu, \rho, .)\|_{\infty}, \quad x \geq 0 .
$$

As (4.12) is a strict inequality then (4.16) is a strict one too if $\|G(0, \mu, \rho, .)\|_{\infty} \neq 0$.

According to $b)$ and $c$ ), there exists $x_{0} \geq 0$ such that: $G\left(0, \mu, \rho, x_{0}\right)=\|G(0, \mu, \rho, .)\|_{\infty}$. This implies $\|G(0, \mu, \rho, .)\|_{\infty}=0$.

Proof of Proposition 4.3. Using the explicit expression of $F_{0}$ and $F_{1}$ ( $c f$. (2.12) and (2.13)), by a straightforward calculation, enables us to infer that both $F_{0}(\theta, \mu, \rho,$.$) and F_{1}(\theta, \mu, \rho,$.$) belong to \mathcal{B}_{\gamma}$ (for a detailed proof, $c f$. [20]).

Due to Lemma 4.4 and (4.15)we may obtain:

$$
\left.F(\theta, \mu, \rho, x)=\sum_{n=0}^{\infty} \Lambda_{\theta}^{n}\left(F_{0}+F_{1}\right)(\theta, \mu, \rho, .)\right)(x)
$$

Because $F_{0}+F_{1} \in \mathcal{B}_{\gamma}$ and $\Lambda_{\theta}$ is a contraction in $\mathcal{B}_{\gamma}$, the serie converges in $\mathcal{B}_{\gamma}$, which directly implies the result.

\section{Remark 4.5.}

1. Under the conditions stated in Proposition 4.3, we have actually proved that $F(\theta, \mu, \rho, x)$ can be approximated by $\sum_{n=0}^{p} \Lambda_{\theta}^{n}\left[\left(F_{0}+F_{1}\right)(\theta, \mu, \rho,).\right](x)$. More precisely:

$$
\left|F(\theta, \mu, \rho, x)-\sum_{n=0}^{p} \Lambda_{\theta}^{n}\left[\left(F_{0}+F_{1}\right)(\theta, \mu, \rho, .)\right](x)\right|<c_{\theta, \gamma}^{p+1} K \mathrm{e}^{-\gamma x},
$$

where $K=\left\|\sum_{n=0}^{\infty} \Lambda_{\theta}^{n}\left[\left(F_{0}+F_{1}\right)(\theta, \mu, \rho, .)\right]\right\|_{\gamma}<\infty$ and $c_{\theta, \gamma}$ is defined by (4.9). 
2. Let us consider the case when the support of $\nu$ is included in $]-\infty, 0]$. $\varphi$ is well defined on ] $-\infty, 0]$ and $r_{\nu}=\infty$. Moreover $K_{x}=L_{x}=0$ and $F_{1}(\theta, \mu, \rho, x)=0$ for any $x \geq 0$. As a result, $(2.10)$ is reduced to:

$$
F(\theta, \mu, \rho, x)=\mathrm{e}^{-\left(c_{+} \alpha_{\theta}\right) x}+\Lambda_{\theta} F(\theta, \mu, \rho, .)(x) .
$$

If either $\theta>0$, or $\theta=0$ and $\mathbb{E}\left(X_{1}\right)<0$, from both (1.25) and (1.26) we can infer the existence of a unique real number $\gamma_{0}(\theta)$ such that:

$$
-\gamma_{0}(\theta)<0 \quad \text { and } \quad \varphi\left(-\gamma_{0}(\theta)\right)=\theta .
$$

A direct (but fastidious !) calculation shows that $x \rightarrow \mathrm{e}^{-\gamma_{0}(\theta) x}$ is a solution of (2.10). For more details see [20]. Hence $F(\theta, \mu, \rho, x)=F(\theta, 0,0, x)=\mathrm{e}^{-\gamma_{0}(\theta) x}$.

\subsection{Few examples of $\nu$ satisfying (2.23)}

We give three classes of measures $\nu$ which satisfy $(H)$ and $(2.23)$ :

a) Let us suppose that $\nu$ has finite exponential moments:

$$
\forall q \in \mathbb{R} \quad \int_{-\infty}^{\infty}\left|\mathrm{e}^{-q y}-1+q y \mathbb{1}_{\{|y|<1\}}\right| \nu(\mathrm{d} y)<\infty
$$

In that case, $\widehat{\nu_{\mid[1, \infty]}}$ and $\varphi$ are holomorphic functions in the whole plane $\mathbb{C}$, then $B_{\nu}=\infty$. Moreover, for any $B>0$ :

$$
\sup _{\operatorname{Re} q \geq-B}\left|\int_{1}^{\infty} \mathrm{e}^{-q y} \nu(\mathrm{d} y)\right| \leq \int_{1}^{\infty} \mathrm{e}^{B y} \nu(\mathrm{d} y)<\infty .
$$

Then (2.23) holds. The condition (4.21) is fulfilled if, for instance, $\nu$ has a compact support.

b) Let $\nu$ be a linear combination of gamma distributions:

$$
\nu(\mathrm{d} y):=\sum_{i=1}^{n} \rho_{i} \mathrm{e}^{-\beta_{i} y} y^{m_{i}} \mathbb{1}_{\{y \geq 0\}} \mathrm{d} y,
$$

where $\rho_{i}>0, \beta_{i}>0$ and $m_{i} \in \mathbb{N}$, for any $i \in\{1,2, \cdots, n\}$.

Since the Laplace transform of $\nu$ is explicit, we obtain immediately its meromorphic extension to the whole plane $\left(B_{\nu}=\infty\right)$ and (2.23). Moreover:

$$
\widehat{\nu}(q)=\int_{0}^{\infty} \mathrm{e}^{-q x} \sum_{i=1}^{n} \rho_{i} \mathrm{e}^{-\beta_{i} x} x^{m_{i}} \mathrm{~d} x=\sum_{i=1}^{n} \rho_{i} \int_{0}^{\infty} x^{m_{i}} \mathrm{e}^{-(q+\beta i) x} \mathrm{~d} x
$$

If we set $y=\left(q+\beta_{i}\right) x$, we have:

$$
\widehat{\nu}(q)=\sum_{i=1}^{n} \frac{\rho_{i}}{\left(q+\beta_{i}\right)^{m_{i}+1}} \int_{0}^{\infty} y^{m_{i}} \mathrm{e}^{-y} \mathrm{~d} y=\sum_{i=1}^{n} \frac{\rho_{i} m_{i} !}{\left(q+\beta_{i}\right)^{m_{i}+1}} .
$$

This implies that $\widehat{\nu}$ resp. $\varphi$ is holomorphic in $\mathbb{C}-\left\{-\beta_{1}, \cdots,-\beta_{n}\right\}$ resp. meromorphic in $\mathbb{C}$.

c) The example above may be generalized as follows:

$$
\nu(\mathrm{d} y):=\phi(y) \mathbb{1}_{\{y \geq 0\}} \mathrm{d} y,
$$

where $\phi \geq 0$, bounded on $\left[0, y_{0}\right]$, and forevery $y \geq y_{0}$ : 


$$
\phi(y):=\rho_{0} \mathrm{e}^{-\beta_{0} y} y^{m_{0}-1}+\sum_{i=1}^{n}\left(\rho_{i} \mathrm{e}^{-\beta_{i} y}+\overline{\rho_{i}} \mathrm{e}^{-\overline{\beta_{i}} y}\right) y^{m_{i}-1}+\mathrm{O}\left(\mathrm{e}^{-\beta_{n+1} y}\right),
$$

with $y_{0} \geq 0, \rho_{0} \geq 0, \beta_{0}>0, \operatorname{Re} \beta_{i}>0, \rho_{i} \in \mathbb{C}^{*}, m_{i} \in \mathbb{N}^{*}$ et $\beta_{n+1} \geq \sup _{1 \leq i \leq n} \operatorname{Re} \beta_{i}$.

Then $\widehat{\nu}$ and $\varphi$ are meromorphic functions in $\left\{q ; \operatorname{Re} q \geq \beta_{n+1}\right\}, B_{\nu}=\bar{\beta}_{n+1}$ and (2.23) holds.

d) If $\nu_{1}$ and $\nu_{2}$ satisfy (2.23), then $\nu=\nu_{1}+\nu_{2}$ satisfies (2.23) too.

\subsection{Calculation of the $C_{i}$ coefficients}

Let us suppose in this section that $(H)$ holds.

a) We claim that $C_{i}(\theta, \mu, \rho,$.$) is a polynomial function. We may assume that -\gamma_{i}(\theta)$ is a zero of $\varphi_{\theta}$ with multiplicity $n_{i}$, and $\widehat{F}(\theta, \mu, \rho, z)$ has the following asymptotic expansion in a neighborhood of $-\gamma_{i}(\theta)$ :

$$
\widehat{F}(\theta, \mu, \rho, z)=\frac{K_{i, n_{i}}(\theta, \mu, \rho)}{\left(z+\gamma_{i}(\theta)\right)^{n_{i}}}+\frac{K_{i, n_{i}-1}(\theta, \mu, \rho)}{\left(z+\gamma_{i}(\theta)\right)^{n_{i}-1}}+\cdots+\frac{K_{i, 1}(\theta, \mu, \rho)}{z+\gamma_{i}(\theta)}+\cdots
$$

Since

$$
\mathrm{e}^{z x}=\mathrm{e}^{-\gamma_{i}(\theta) x}\left(1+\left(z+\gamma_{i}(\theta)\right) x+\frac{\left(z+\gamma_{i}(\theta)\right)^{2}}{2 !} x^{2}+\cdots\right) .
$$

then relations (3.80) and (3.81) imply that:

$$
C_{i}(\theta, \mu, \rho, x)=\frac{K_{i, n_{i}}(\theta, \mu, \rho)}{\left(n_{i}-1\right) !} x^{n_{i}-1}+\frac{K_{i, n_{i}-1}(\theta, \mu, \rho)}{\left(n_{i}-2\right) !} x^{n_{i}-2}+\cdots+K_{i, 1}(\theta, \mu, \rho) .
$$

b) We suppose in this item that $-\gamma_{i}(\theta)$ is a single zero of $\varphi_{\theta}=\varphi-\theta$. Then $C_{i}(\theta, \mu, \rho, x)$ does not depend on $x$. Furthermore $C_{i}(\theta, \mu, \rho, x)$ is given by the following:

$$
C_{i}(\theta, \mu, \rho)=\operatorname{Res}\left(\widehat{F}(\theta, \mu, \rho, z) ;-\gamma_{i}(\theta)\right) .
$$

Note, that in this case, according to $(2.17),-\gamma_{i}(\theta)$ is a single pole of $\widehat{F}(\theta, \mu, \rho, \cdot)$.

i) When the real part of $-\gamma_{i}(\theta)$ is bigger than $-r_{\nu}, C_{i}(\theta, \mu, \rho)$ can be determined as follows:

$$
\begin{aligned}
C_{i}(\theta, \mu, \rho)= & \frac{1}{\varphi^{\prime}\left(-\gamma_{i}(\theta)\right)}\left[\frac{-\gamma_{i}(\theta)-\gamma_{0}^{*}(\theta)}{2}\right. \\
& +\int_{0}^{\infty}\left[\frac{\mathrm{e}^{\left(\gamma_{i}(\theta)-\rho\right) y}-\mathrm{e}^{-\mu y}}{-\gamma_{i}(\theta)+\rho-\mu}-\frac{\mathrm{e}^{-\left(\gamma_{0}^{*}(\theta)+\rho\right) y}-\mathrm{e}^{-\mu y}}{\gamma_{0}^{*}(\theta)+\rho-\mu}\right] \nu(\mathrm{d} y) \\
& \left.+R F(\theta, \mu, \rho, .)\left(-\gamma_{i}(\theta)\right)-R F(\theta, \mu, \rho, .)\left(\gamma_{0}^{*}(\theta)\right)\right],
\end{aligned}
$$

where it is supposed that $\frac{\mathrm{e}^{a y}-1}{a}=y$ if $a=0$.

It has to be observed that if the support of $\nu$ is included in $] 0, \infty[$, then the formula above reduces to the two first lines, since $R F(\theta, \mu, \rho, \cdot)=0$. What is more, if $\theta=\mu=\rho=0$ then

$$
C_{i}(0,0,0)=\left\{\begin{array}{cc}
\frac{\varphi^{\prime}(0)}{\varphi^{\prime}\left(-\gamma_{i}(0)\right)} & \text { if } \mathbb{E}\left(X_{1}\right)<0 \\
1 & \text { if } i=0 \text { and } \mathbb{E}\left(X_{1}\right) \geq 0 \\
0 & \text { if } i \geq 1 \text { and } \mathbb{E}\left(X_{1}\right) \geq 0
\end{array}\right.
$$

Considering $i=0$ in (4.33) allows to recover the result given in [5]. 
ii) As for the case $\operatorname{Re}\left(-\gamma_{i}(\theta)\right)<-r_{\nu}$, the previous $\nu$-integrals and $\varphi$, have to be replaced by their meromorphic extensions.

c) In this item we can focus on $C_{0}(\theta, \mu, \rho)$, which is the dominent term in (2.25).

i) When $\theta>0$ or $\theta=0$ and $\mathbb{E}\left(X_{1}\right) \neq 0$, then $-\gamma_{0}(\theta)$ is a single zero of $\varphi_{\theta}$ and $-\gamma_{0}(\theta)>-r_{\nu}$. Therefore $C_{0}(\theta, \mu, \rho)$ is given by $(4.32)$ with $i=0$. Let us recall that when $\theta=0$ and $\mathbb{E}\left(X_{1}\right)<0\left(\operatorname{resp} . \mathbb{E}\left(X_{1}\right)>0\right)$, then $\gamma_{0}^{*}(0)=0\left(\right.$ resp. $\left.\gamma_{0}(0)=0\right)$.

ii) In the case $\mathbb{E}\left(X_{1}\right)=0$, then $\gamma_{0}(0)=\gamma_{0}^{*}(0)=0$ is a double zero of $\varphi$, but a simple pole of $\widehat{F}(\theta, \mu, \rho,$.$) .$ Thus, a direct calculation shows:

$$
\begin{aligned}
C_{0}(0, \mu, \rho)= & \frac{1}{\varphi^{\prime \prime}(0)}\left(1-\frac{2}{(\rho-\mu)^{2}} \int_{0}^{\infty} \mathrm{e}^{-\rho y}\left(1-\mathrm{e}^{(\rho-\mu) y}+(\rho-\mu) y\right) \nu(\mathrm{d} y)\right. \\
& \left.-2 \int_{-\infty}^{0} \nu(\mathrm{d} y) \int_{0}^{-y}(y+b) F(0, \mu, \rho, b) \mathrm{d} b\right) .
\end{aligned}
$$

In particular:

$$
\begin{aligned}
C_{0}(0,0,0) & =\frac{1}{\varphi^{\prime \prime}(0)}\left(1+\int_{0}^{\infty} y^{2} \nu(\mathrm{d} y)-2 \int_{-\infty}^{0} \nu(\mathrm{d} y) \int_{0}^{-y}(y+b) F(0,0, b) \mathrm{d} b\right) \\
& =1-\frac{2}{\varphi^{\prime \prime}(0)} \int_{-\infty}^{0} \nu(\mathrm{d} y) \int_{0}^{-y}(y+b) F(0,0,0, b) \mathrm{d} b
\end{aligned}
$$

iii) The constant $C_{0}(\theta, \mu, \rho)$ is positive because $\mu \rightarrow \widehat{F}\left(\theta, \mu, q-\gamma_{0}(\theta)\right)$ is decreasing and

$$
\lim _{\mu \rightarrow \infty} C_{0}(\theta, \mu, \rho)=\left\{\begin{array}{cl}
\frac{1}{\varphi^{\prime \prime}(0)}>0 & \text { if } \theta=0 \text { and } \mathbb{E}\left(X_{1}\right)=0 \\
-\frac{\gamma_{0}(\theta)+\gamma_{0}^{*}(\theta)}{2 \varphi^{\prime}\left(-\gamma_{0}(\theta)\right)}>0 & \text { otherwise. }
\end{array}\right.
$$

\subsection{Girsanov transformation}

Let $\left(X_{t}, t \geq 0\right)$ be a Lévy process. It is well known that there is a family of probability measures $\left(\mathbb{P}^{(\lambda)}, 0 \leq \lambda \leq \gamma\right)$ so that, under $\mathbb{P}^{(\lambda)},\left(X_{t}, t \geq 0\right)$ is still a Lévy process and:

$$
\mathbb{P}^{(\lambda)}\left(X_{t} \in \mathrm{d} x\right)=\mathrm{e}^{\lambda x} \mathrm{e}^{-t \varphi(-\lambda)} \mathbb{P}\left(X_{t} \in \mathrm{d} x\right)
$$

Consequently $\varphi^{(\lambda)}(q)=\varphi(q-\lambda)-\varphi(-\lambda)$, where $\varphi^{(\lambda)}$ is associated with $\left(X_{t}, t \geq 0\right)$ under $\mathbb{P}^{(\lambda)}$. Under $(H)$, there exists $\lambda$ such that $\varphi(-\lambda)=\theta$ and $\varphi^{\prime}(0) \varphi^{\prime}(-\lambda)<0$. Since $\mathbb{E}\left(X_{1}\right)=-\varphi^{\prime}(0)$, and $\mathrm{e}^{(\lambda)}\left(X_{1}\right)=-\varphi^{\prime(\lambda)}(0)=$ $-\varphi^{\prime}(-\lambda)$, then $\mathbb{E}\left(X_{1}\right) \mathrm{e}^{(\lambda)}\left(X_{1}\right)<0$. This trick allows to only consider the case $\mathbb{E}\left(X_{1}\right)>0$ (or $\left.\mathbb{E}\left(X_{1}\right)<0\right)$, and then simplify the proofs of Theorems 2.1 and 2.3 and the result given in subsection 4.1 .

\subsection{Wiener-Hopf factorization}

Let us recall the Wiener-Hopf decomposition ( $c f$. [1], p. 165): for any $\theta>0$, we have:

$$
\frac{\theta}{\theta+\varphi(-q)}=\psi_{\theta}^{+}(q) \psi_{\theta}^{-}(q)
$$

where

$$
\psi_{\theta}^{+}(q):=\mathbb{E}\left(\mathrm{e}^{i q S_{\tau_{\theta}}}\right) \quad, \quad \psi_{\theta}^{-}(q):=\mathbb{E}\left(\mathrm{e}^{i q\left(S_{\tau_{\theta}}-X_{\tau_{\theta}}\right)}\right)
$$


and $\tau_{\theta}$ is an exponential r. v. with parameter $\theta$, independent from process $\left(X_{t}, t \geq 0\right)$ and $S_{t}:=\sup _{s \leq t} X_{s}$. Since:

$$
\mathbb{P}\left(S_{\tau_{\theta}}>a\right)=\mathbb{P}\left(T_{a}<\tau_{\theta}\right)=\mathbb{E}\left(\mathrm{e}^{-\theta T_{a}}\right)=F(\theta, 0,0, a),
$$

it is easy to deduce the following identity:

$$
\psi_{\theta}^{+}(q)=1+i q \widehat{F}(\theta, 0,0, i q) .
$$

Equation (2.17) implies that the Wiener-Hopf factor $\psi_{\theta}^{+}$verifies a functional equation. In particular, if $\nu(]-$ $\infty, 0])=0$, combining equations (2.18) and (4.40) an explicit form of $\psi_{\theta}^{+}(q)$ may be obtained. Due to (4.37), $\psi_{\theta}^{-}(q)$ is also explicit.

\subsection{Proof of Proposition 2.7}

1. It is clear that $\varphi_{\theta}$ is holomorphic in $\left\{q \in \mathbb{C} ; \operatorname{Re} q>-r_{\nu}\right\}$ and the only real zeros of $\varphi_{\theta}$ in this domain are $-\gamma_{0}(\theta)$ and $\gamma_{0}^{*}(\theta)$.

2. We claim that the complex zeros of $\varphi_{\theta}$ in $\left\{q \in \mathbb{C} ;-\gamma_{0}(\theta) \leq \operatorname{Re} q \leq \gamma_{0}^{*}(\theta)\right\}$ are $-\gamma_{0}(\theta)$ and $\gamma_{0}^{*}(\theta)$.

a) Suppose first that $-\gamma_{0}(\theta)<\operatorname{Re} q<\gamma_{0}^{*}(\theta)$. We have:

$$
\left|\mathrm{e}^{\varphi_{\theta}(q)}\right|=\left|\mathbb{E}\left(\mathrm{e}^{-q X_{1}-\theta}\right)\right| \leq \mathbb{E}\left(\mathrm{e}^{-\operatorname{Re} q X_{1}-\theta}\right)=\mathrm{e}^{\varphi_{\theta}(\operatorname{Re} q)}<1,
$$

since $\varphi_{\theta}<0$ on $]-\gamma_{0}(\theta), \gamma_{0}^{*}(\theta)\left[\right.$. Then $\varphi_{\theta}(q) \neq 0$.

b) Let $q=-\gamma_{0}(\theta)+i b, b \in \mathbb{R}$. We compute $\varphi_{\theta}(q)$ :

$$
\begin{aligned}
\varphi_{\theta}(q)= & \varphi_{\theta}\left(-\gamma_{0}(\theta)\right)-\frac{b^{2}}{2}+\int_{-\infty}^{\infty} \mathrm{e}^{\gamma_{0}(\theta) y}(\cos (b y)-1) \nu(\mathrm{d} y) \\
& +i\left(-b \gamma_{0}(\theta)+c b-\int_{-\infty}^{\infty}\left(\mathrm{e}^{\gamma_{0}(\theta) y} \sin (b y)-b y \mathbb{1}_{\{|y|<1\}}\right) \nu(\mathrm{d} y)\right) .
\end{aligned}
$$

But $\varphi_{\theta}\left(-\gamma_{0}(\theta)\right)=0$, then $\operatorname{Re}\left(\varphi_{\theta}(q)\right) \leq-\frac{b^{2}}{2}$. Consequently $\varphi_{\theta}(q)=0$ iff $b=0$.

c) The same reasoning applies to the case $q=-\gamma_{0}^{*}(\theta)+i b$.

3. Let us prove that $\varphi_{\theta}$ has a finite number of zeros in the strip $\left\{q \in \mathbb{C} ;-B \leq \operatorname{Re} q \leq \beta_{\theta}\right\}$, for some $\beta_{\theta}$ in ] $0, \gamma_{0}^{*}(\theta)$ [. Proposition 2.7 is a consequence of both items 1. and 2. as described above and of the following properties:

(i) According to (2.23) there is $R>R_{0}>0, k>0$ so that:

$$
\left|\varphi_{\theta}(q)\right| \geq k\left|q^{2}\right| \text { for any } q \text { such that }-B \leq \operatorname{Re} q \leq 0,|\operatorname{Im} q|>R .
$$

As a result, $\varphi_{\theta}(q) \neq 0$.

(ii) The function $\varphi_{\theta}$ is meromorphic in $\{q \in \mathbb{C} ; \operatorname{Re} q>-B\}$ and therefore admits at most a finite number of poles in the compact domain $\{q \in \mathbb{C} ;-B \leq \operatorname{Re} q \leq 0,|\operatorname{Im} q| \leq R\}$.

Acknowledgements. We wish to thank the referee for carefully reading the manuscript and making useful suggestions. 


\section{REFERENCES}

[1] J. Bertoin, Lévy processes, Cambridge Tracts in Mathematics, vol. 121. Cambridge University Press, Cambridge (1996).

[2] J. Bertoin and R.A. Doney, Cramér's estimate for Lévy processes. Statist. Probab. Lett. 21 (1994) 363-365.

[3] H. Cramér, Collective risk theory: A survey of the theory from the point of view of the theory of stochastic processes. Skandia Insurance Company, Stockholm, (1955). Reprinted from the Jubilee Volume of Försäkringsaktiebolaget Skandia.

[4] H. Cramér, On the mathematical Theory of Risk. Skandia Jubilee Volume, Stockholm (1930).

[5] R.A. Doney, Hitting probabilities for spectrally positive Lévy processes. J. London Math. Soc. 44 (1991) 566-576.

[6] R.A. Doney and A.E. Kyprianou, Overshoots and undershoots of Lévy processes. Ann. Appl. Probab. 16 (2006) 91-106.

[7] R.A. Doney and R.A. Maller. Stability of the overshoot for Lévy processes. Ann. Probab. 30 (2002) 188-212.

[8] F. Dufresne and H.U. Gerber, Risk theory for the compound Poisson process that is perturbed by diffusion. Insurance Math. Econom. 10 (1991) 51-59.

[9] I.S. Gradshteyn and I.M. Ryzhik, Table of integrals, series, and products. Academic Press [Harcourt Brace Jovanovich Publishers], New York (1980). Corrected and enlarged edition edited by Alan Jeffrey, Incorporating the fourth edition edited by Yu. V. Geronimus [Yu. V. Geronimus] and M. Yu. Tseytlin [M. Yu. Tseľtlin], Translated from Russian.

[10] P.S. Griffin and R.A. Maller, On the rate of growth of the overshoot and the maximum partial sum. Adv. in Appl. Probab. 30 (1998) 181-196.

[11] A. Gut, Stopped random walks, Applied Probability, vol. 5, A Series of the Applied Probability Trust. Springer-Verlag, New York, (1988). Limit theorems and applications.

[12] I. Karatzas and S.E. Shreve. Brownian motion and stochastic calculus, Graduate Texts in Mathematics, vol.113. SpringerVerlag, New York, second edition (1991).

[13] A.E. Kyprianou, Introductory lectures on fluctuations of Lévy processes with applications. Universitext. Springer-Verlag, Berlin (2006).

[14] N.N. Lebedev, Special functions and their applications. Dover Publications Inc., New York (1972). Revised edition, translated from the Russian and edited by Richard A. Silverman, Unabridged and corrected republication.

[15] M. Loève, Probability theory. II. Springer-Verlag, New York, fourth edition (1978). Graduate Texts in Mathematics, Vol. 46.

[16] F. Lundberg, I- Approximerad Framställning av Sannolikhetsfunktionen. II-Aterförsäkering av Kollectivrisker. Almqvist and Wiksell, Uppsala (1903).

[17] T. Rolski, H. Schmidli, V. Schmidt and J. Teugels, Stochastic processes for insurance and finance. Wiley Series in Probability and Statistics. John Wiley \& Sons Ltd., Chichester (1999).

[18] K. Sato, Lévy processes and infinitely divisible distributions, volume 68 of Cambridge Studies in Advanced Mathematics. Cambridge University Press, Cambridge, (1999). Translated from the 1990 Japanese original, Revised by the author.

[19] A.G. Sveshnikov and A.N. Tikhonov, The theory of functions of a complex variable. "Mir", Moscow (1982). Translated from the Russian by George Yankovsky [G. Yankovskiı̄].

[20] A. Volpi, Processus associés à l'équation de diffusion rapide; Étude asymptotique du temps de ruine et de l'overshoot. Univ. Henri Poincaré, Nancy I, Vandoeuvre les Nancy (2003). Thèse. 